\title{
A numerical manifold method model for analyzing fully coupled hydro-mechanical processes in porous rock masses with discrete fractures
}

\author{
Mengsu $\mathrm{Hu}^{1,2}$ \\ ${ }^{1}$ College of Civil and Transportation Engineering, Hohai University, \\ Nanjing, 210098, China \\ ${ }^{2}$ Energy Geosciences Division, Lawrence Berkeley National Laboratory, \\ Berkeley, CA 94720, USA \\ Email address: mengsuhu@lbl.gov \\ Jonny Rutqvist ${ }^{2}$ \\ ${ }^{2}$ Energy Geosciences Division, Lawrence Berkeley National Laboratory, \\ Berkeley, CA 94720, USA \\ Email address: jrutqvist@lbl.gov
Yuan Wang ${ }^{1,2, *}$ ( Corresponding author)
${ }^{1}$ College of Civil and Transportation Engineering, Hohai University, Nanjing, 210098, China
2 Energy Geosciences Division, Lawrence Berkeley National Laboratory,
Berkeley, CA 94720, USA
Email address: wangyuanhhu@163.com or wangyuan@hhu.edu.cn
Telephone Number: 86-25-83787163




\title{
A numerical manifold method model for analyzing fully coupled
}

\section{hydro-mechanical processes in porous rock masses with discrete}

\author{
fractures \\ Mengsu $\mathrm{Hu}^{1,2}$, Jonny Rutqvist ${ }^{2}$, Yuan Wang ${ }^{1,2, *}$ \\ ${ }^{1}$ College of Civil and Transportation Engineering, Hohai University, \\ Nanjing, 210098, China \\ ${ }^{2}$ Energy Geosciences Division, Lawrence Berkeley National Laboratory, Berkeley, \\ CA 94720, USA
}

\begin{abstract}
In this study, a numerical manifold method (NMM) model was developed for fully coupled analysis of hydro-mechanical (HM) processes in porous rock masses with discrete fractures. Using an NMM two-cover-mesh system of mathematical and physical covers, fractures are conveniently discretized by dividing the mathematical cover along fracture traces to physical cover, resulting in a discontinuous model on a non-conforming mesh. In this model, discrete fracture deformation (e.g. open and slip) and fracture fluid flow within a permeable and deformable porous rock matrix are rigorously considered. For porous rock, direct pore-volume coupling was modeled based on an energy-work scheme. For mechanical analysis of fractures, a fracture constitutive model for mechanically open states was introduced. For fluid flow in fractures, both along-fracture and normal-to-fracture fluid flow are modeled without introducing additional degrees of freedom. When the mechanical aperture of a fracture is changing, its hydraulic aperture and hydraulic conductivity is updated. At the same time, under the effect of coupled deformation and fluid flow, the contact state may dynamically change, and the corresponding contact constraint is updated each time step. Therefore, indirect coupling is realized under stringent considerations of coupled HM effects and fracture constitutive behavior transfer dynamically. To verify the new model, examples involving deformable porous media containing a single and two sets of fractures were designed, showing good accuracy. Last, the model was applied to analyze coupled HM behavior of fractured porous rock domains with complex fracture networks under effects of loading and injection.
\end{abstract}

Keywords: discrete fractures, full coupling, direct and indirect couplings, numerical manifold method, non-conforming mesh

\section{Introduction}

Coupled Hydro-mechanical (HM) processes are significant in subsurface engineering activities, such as oil and gas extraction, geothermal energy and nuclear waste disposal [1]. In the geological media, thin (microns to millimeters) fractures may ubiquitously exist and be arbitrarily oriented and complexly intersected. Therefore, 
two types of hydro-mechanical couplings prevails, i.e., direct and indirect couplings [1]. Direct coupling via pore-volume interaction means that fluid pressure changes instantaneously induce deformation while mechanical volume changes instantaneously induce fluid pressure changes. This pore-volume interaction can be described by Biot's equations [2,3]. Indirect coupling refers to indirect interaction between mechanical and hydraulic fields through changes in their material properties $[1,4]$ and is usually represented by constitutive functions based on laboratory data or in situ experiments [5-7]. A number of numerical models have been developed for analyzing coupled hydro-mechanical processes in fractured rock. They can be categorized into continuous models such as equivalent continuum and dual-continuum models, discontinuous models, and their hybrid. As the arbitrarily oriented fractures may influence the deformation and fluid distribution in a complex way that cannot be simplified as a continuum, numerical modeling considering discrete fractures with full coupling capability is of great importance.

It is quite challenging to accurately and efficiently represent the complexly intersected fractures under coupled HM effects. Many finite element codes were developed for analyzing coupled HM behavior of fractured rock at various levels of sophistication [8-12], and were applied in analysis of nuclear waste disposal and geothermal energy. However, difficulty of explicit representation of fractures in FEM limits their application to geological media involving complex fracture networks. On the other hand, discontinuous models such as UDEC [13], 3DEC [14], and Discontinuous Deformation Analysis (DDA) were developed [15-16], but were limited by the assumption that the rock matrix is impermeable. Later models with non-conforming mesh based on extended [17] and enriched [18] FEM were developed, but the required additional junction functions for intersections of fractures limit their applicability (An et al. [19]). Due to aforementioned limitations of the existing models, new models that can rigorously model coupled hydro-mechanical processes in porous rock masses containing complex fracture networks are desired.

The numerical manifold method (NMM, [20-21]) appears to be a promising method for this multi- field problem requiring sufficiently good representation for both continuous and discontinuous media. NMM is based on the theory of mathematical manifolds. The numerical meshes of NMM consist of mathematical cover and physical cover. The mathematical cover overlay the entire material domain and the physical cover are divided from the mathematical covers by boundaries and discontinuities. Therefore, the mathematical cover determine the approximation precision by its mesh density and the physical cover define the integration domain. Both continuous and discontinuous problems can be solved in a unified method. In the past two decades, NMM has been successfully applied to mechanics analysis of both continuous and discontinuous geologic media [22-25]. Later, models for fluid flow were developed, such as for analysis of free-surface flow [26, 27], flow in heterogeneous media [28], and in porous rock with discrete fractures [29-30]. For coupled HM problems, an NMM model for consolidation under dynamic loading [31] in porous media was developed. Most recently, the authors [32] developed a model 
for dominant fractures involving both direct and indirect HM couplings, laying the basis for this study.

In this study, we extend the NMM model for fluid flow in fractured media ([29]) to considering mechanical and coupled HM effects, and develop a fully coupled HM model for porous rock with discrete fractures. We first provide a mathematical statement of the problem and related governing equations in Section 2. Then we introduce the fundamentals of NMM, including the definitions in NMM and its non-conforming mesh generation in Section 3. In Section 4, a fully coupled HM model for fractured rock is developed. This model considers fractures mechanical open, closed and sliding states, and fluid flow along and normal to fractures under effects of direct and indirect couplings. Finally in Section 5 we present several examples and demonstrate the new model's capability to problems involving complex discrete fractures.

\section{Governing Equations}

The pore-volume coupling in porous media, i.e., the direct coupling can be described by Biot's equations [3]:

$$
\begin{gathered}
\nabla \boldsymbol{\sigma}+\mathbf{f} \quad \boldsymbol{\theta} \\
\nabla \cdot \mathbf{v}+\alpha \frac{\partial \varepsilon_{v}}{\partial t}+\frac{\gamma}{M} \frac{\partial h}{\partial t}=0
\end{gathered}
$$

where $\boldsymbol{\sigma}$ is the total stress tensor, $\mathbf{f}$ is the body force vector, $\mathbf{v}$ is the fluid velocity vector, $\alpha$ is the Biot coefficient (usually ranges between 0 and 1 ), $\varepsilon_{v}$ is the volumetric strain of the porous media, $M$ is Biot's modulus, and $h$ is the fluid hydraulic head, as the sum of fluid pressure head $p$ and the head associated with elevation. Equations (1) and (2), coupled through fluid pressure head $p$ and volumetric strain $\varepsilon_{v}$, represent the mechanical equilibrium and fluid mass conservation, respectively. The total stress $\boldsymbol{\sigma}$, effective stress $\boldsymbol{\sigma}^{\prime}$ and pressure head $p$ follow a modified Terzaghi's effective stress law:

$$
\boldsymbol{\sigma}=\boldsymbol{\sigma}^{\prime}-\mathbf{m} \alpha \gamma p
$$

where $\mathbf{m}=[1,1,1,0,0,0]^{\top}$ for $3 D$ analysis and $\mathbf{m}=[1,1,0]^{\top}$ for $2 D$ analysis.

If the porous media is linear elastic, it satisfies:

$$
\boldsymbol{\sigma}^{\prime}=\mathbf{E} \boldsymbol{\varepsilon}
$$

where $\mathbf{E}$ is the elastic constitutive tensor and $\boldsymbol{\varepsilon}$ is the strain tensor. With small-deformation assumption, we have:

$$
\varepsilon=\mathbf{A u}
$$

where $\mathbf{A}$ is the strain-displacement matrix and $\mathbf{u}$ is the displacement vector. Fluid flow in porous media satisfies Darcy`s law:

$$
\mathbf{v}=-\operatorname{Kgrad}(h)
$$

where $\mathbf{K}$ is the tensor of permeability coefficient.

Deformation of thin fractures is modeled using the concept of fracture normal stiffness $\left(k_{n}\right)$ and shear stiffness $\left(k_{s}\right)$, expressed as: 


$$
\left(\begin{array}{l}
d \sigma_{n}^{\prime} \\
d \sigma_{s}^{\prime}
\end{array}\right)=\left(\begin{array}{ll}
k_{n} & 0 \\
0 & k_{s}
\end{array}\right)\left(\begin{array}{l}
d v_{n} \\
d v_{s}
\end{array}\right)
$$

Fluid flow in fractures also satisfies the Darcy`s flow:

$$
\left(\begin{array}{c}
v_{s}^{f} \\
v_{n}^{f}
\end{array}\right)=-\left(\begin{array}{ll}
k_{f} & 0 \\
0 & k_{f n}
\end{array}\right)\left(\begin{array}{c}
\frac{\partial h^{f}}{\partial s} \\
\frac{\partial h^{f}}{\partial n}
\end{array}\right)
$$

where $k_{f}$ and $k_{f n}$ are the fracture hydraulic conductivities along and normal to the fracture. $k_{f}$ is a function of the fracture hydraulic aperture $b_{h}$, expressed according to Rutqvist et al. [5]:

$$
k_{f}=\frac{b_{h}^{2} \rho g}{12 \mu}
$$

where $\rho$ and $\mu$ are the fluid density and dynamic viscosity, $g$ is the gravitational acceleration respectively. In this study, $k_{f n}$ is assigned the same value as the $k_{f}$, which is adequate at least in cases when fracture permeability is much larger than the matrix permeability. The hydraulic aperture $b_{h}$ is assumed a function of mechanical aperture $b_{m}[5]$ :

$$
b_{h}=b_{h r}+f b_{m}
$$

where $b_{h r}$ is the residual hydraulic aperture denoting a fracture conductivity while it is mechanically closed, and $f$ is a factor representing the deviation of flow in a natural rough fracture from the ideal parallel smooth fracture.

The boundary and initial conditions for coupled HM processes in the fractured porous media are: $\mathbf{u}=\overline{\mathbf{u}}$ on $\Gamma_{\mathrm{u}}$ as a displacement boundary condition, $\boldsymbol{\sigma} \cdot \mathbf{n}=\overline{\mathbf{t}}$ on $\Gamma_{\mathrm{t}}$ as a traction boundary condition, $\mathbf{p}_{p}=\overline{\mathbf{p}}_{p}$ on $\Gamma_{\mathrm{p}}$ as a flow Dirichlet boundary condition, $\mathbf{v} \cdot \mathbf{n}=\overline{\mathbf{q}}$ on $\Gamma_{\mathrm{v}}$ as a flow Neumann boundary condition, and $\mathbf{u}(\mathbf{x}, 0)=\mathbf{u}_{\mathbf{0}}(\mathbf{x})$, $\boldsymbol{\sigma}(\mathbf{x}, 0)=\sigma_{\mathbf{0}}(\mathbf{x}), \quad \mathbf{p}_{p}(\mathbf{x}, 0)=\mathbf{p}_{\mathbf{0}}(\mathbf{x})$ in $\Omega$ as initial conditions of displacement, stress and fluid pressure, respectively.

\section{Fundamentals of NMM}

The NMM (Shi, [20]) is based on the concept of "manifold" in topology. In NMM, independent mathematical cover and physical cover are defined. A mathematical cover are a set of connected patches that cover the whole material domain [33]. Regular meshes such as finite difference grids and finite elements can be used as mathematical patches. The physical patches are mathematical patches divided by boundaries and discontinuities, determining the integration fields. The union of all the physical patches forms a physical cover. On each physical patch, a local function is 
assigned, for example constant, linear function or series. Specifically, if a patch function is constant, NMM can be simplified as FEM. The weighted average of local patch functions forms the global approximation. Therefore, the NMM provides a flexible and general approach to include continuous and discontinuous methods in a unified form. A detailed comparison of NMM to FEM and DDA were made by the authors in [29]. In this study, we use triangles to generate the mathematical cover. Herein we briefly introduce how mathematical and physical covers are defined in the triangular mesh. As shown in Figure 1, all the triangles sharing one certain node (or 'star', such as $P_{1}, P_{2}$, and $P_{3}$ ) form a hexagonal mathematical patch. The physical patches $\left(\mathrm{P}_{1}{ }^{1}\right.$ and $\mathrm{P}_{1}{ }^{2}, \mathrm{P}_{2}{ }^{1}$ and $\mathrm{P}_{2}{ }^{2}$, and $\mathrm{P}_{3}{ }^{1}$ and $\left.\mathrm{P}_{3}{ }^{2}\right)$ are divided from the mathematical patches by boundaries and fractures. The overlapping areas of physical patches such as $\mathrm{P}_{1}{ }^{1} \mathrm{P}_{2}{ }^{1} \mathrm{P}_{3}{ }^{1}$ and $\mathrm{P}_{1}{ }^{2} \mathrm{P}_{2}{ }^{2} \mathrm{P}_{3}{ }^{2}$ are defined as elements. In this study, we focus on problems involving a network of densely intersected fractures that do not propagate. Therefore, we don't consider singular physical patches associated with fracture tips. Detailed work on such a problem for singular patches especially for problems involving fracture propagation can be found in [23].
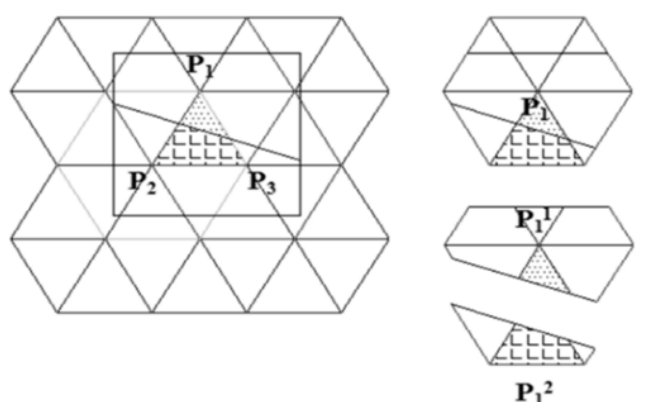

$\mathbf{P}_{1}^{2}$
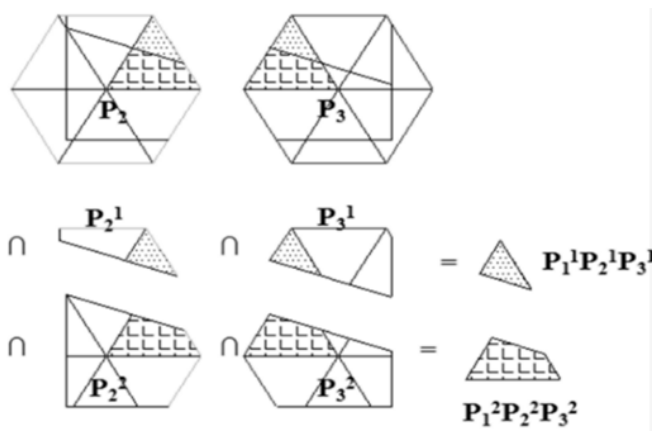

Figure 1 Schematic of a fractured domain discretized in NMM

The global approximations of displacement and pressure head on an element are the weighted average of patch functions:

$$
\begin{gathered}
u=\mathbf{w}_{\mathbf{u}}{ }^{\mathbf{T}} \mathbf{u}_{\mathbf{p c}} \\
p=\mathbf{w}_{\mathbf{p}}{ }^{\mathbf{T}} \mathbf{p}_{\mathbf{p c}}
\end{gathered}
$$

where $u$ and $p$ are the displacement and pressure head on a certain element, $\mathbf{u}_{\mathrm{pc}}$ and $\mathbf{p}_{\mathrm{pc}}$ are the vectors of patch functions of displacement and pressure head, $\mathbf{w}_{\mathbf{u}}$ and $\mathbf{w}_{\mathbf{p}}$ are the vectors of weight functions of patch functions $\mathbf{u}_{\mathrm{pc}}$ and $\mathbf{p}_{\mathrm{pc}}$ on this element. For an individual physical patch $i$, we have

$$
\begin{cases}w_{u}^{i}(x, y)>0, w_{p}^{i}(x, y)>0 & (x, y) \in U_{i} \\ w_{u}^{i}(x, y)=0, w_{p}^{i}(x, y)=0 & (x, y) \notin U_{i}\end{cases}
$$

where $U_{i}$ is the geometric range of physical patch $i$.

The patch functions $\mathbf{u}_{\mathbf{p c}}$ and $\mathbf{p}_{\mathbf{p c}}$ can be a series of any order:

$$
\mathbf{u}_{\mathrm{pc}}=\mathbf{f}^{\mathrm{T}} \mathbf{D}
$$




$$
\mathbf{p}_{\mathbf{p c}}=\mathbf{S}^{\mathbf{T}} \mathbf{P}
$$

where $\mathbf{D}$ and $\mathbf{P}$ are the degrees of freedom (DOFs) to be solved in mechanical and fluid flow fields, respectively, and $\mathbf{f}$ and $\mathbf{s}$ are the coefficients of $\mathbf{D}$ and $\mathbf{P}$. For $2 \mathrm{D}$ analysis, $\mathbf{f}$ and $\mathbf{s}$ are the subsets of vector $\left(1, x, y, x^{2}, y^{2}, x y, \ldots \ldots\right)^{\top}$. In this study, linear weight functions and constant patch functions are used for both mechanical and fluid flow analysis.

The non-conforming mesh with independent mathematical and physical covers for a discrete-fractured media is generated by using an algorithm named tree cutting. The mechanism and implementation of tree cutting algorithm was introduced by Shi [33] and the two methods to represent fractures in permeable rock masses were introduced by the authors [29] in detail. By tree cutting, the intersecting discrete fractures and boundaries divide the mathematical cover into physical cover and thereafter form the non-conforming mesh, as shown in Figure 2.

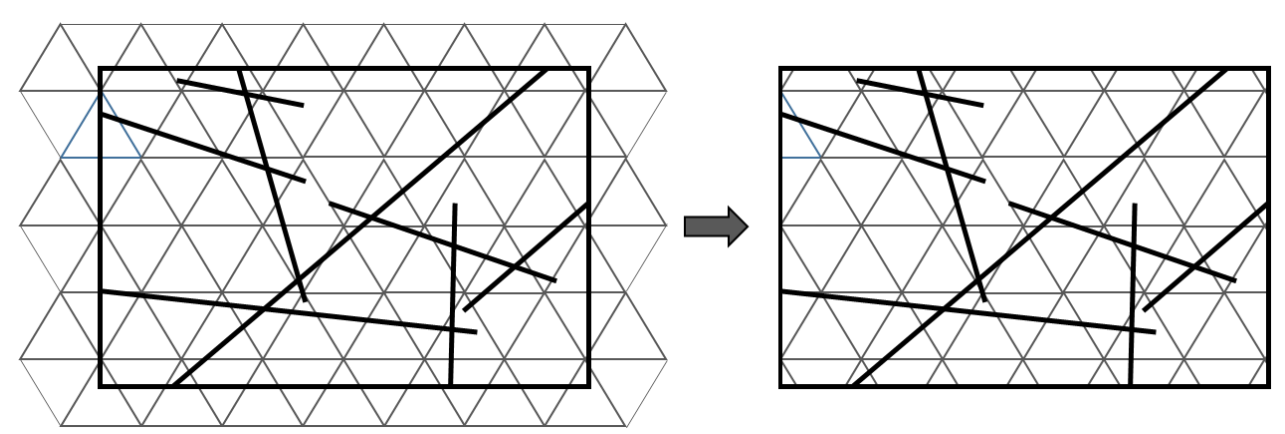

Figure 2 Generated non-conforming mesh

\section{Fully coupled HM model for porous rock masses with discrete}

\section{fractures}

With the tree-cutting algorithm, we are able to generate the physical cover and elements in NMM divided by discrete fractures, boundaries and triangular mesh lines. In this section, we will first introduce an energy-work based scheme for direct coupling (Section 4.1). As fractures are represented by the surfaces of the divided physical cover, we extend the original NMM formulation [20] to consider open, closed and sliding states of fractures (Section 4.2) and use a recently developed discontinuous model [29] for fluid flow in fractures (Section 4.3). Indirect coupling associated with the discrete fractures is realized by changing fractures' hydraulic properties each time step.

\subsection{Energy-work based scheme for direct coupling in porous rock}

The direct coupling in terms of pore-volume effect is modeled based on an energy-work concept, which was previously developed and used for porous rock with dominant fractures [32]. For mechanical analysis developed by Shi [20], the total potential energy associated with each component of dynamic/static mechanical 
processes, possibly involving discontinuous and large deformation was considered. Those energy terms include the energy associated with strain energy, initial stress, point loading, surface loading, body loading and displacement boundary condition. For fluid flow analysis, the authors [26] developed an energy-work seepage model to establish all the work and energy associated with fluid flow in porous media and a Lagrange multiplier method (LMM) for heterogeneous flow [28]. The energy associated with fluid flow in porous media include that associated with domain flow in porous media, fluid gravity, Dirichlet, Neumman and material boundary conditions. Later, the authors extended the energy-work seepage model to conduct coupled HM analysis [32]. The mechanical and fluid flow fields are linked by "work" to consider the pore-volume interaction, which is consistent with the Biot's equations for porous media. This work representing fluid-solid interactions includes the work by the fluid flow on solid deformation, and the work by solid deformation on fluid flow in terms of solid deformation effect on fluid mass balance.

\subsection{Mechanical model of discrete fractures in open, closed and sliding states}

The mechanical analysis for discrete fractures is based on the contact theory developed by Shi [20], including contact detection, contact enforcement and open-close iteration. Contact detection is to detect when and where contact occurs among elements divided by discontinuities. In the current study, each fracture is represented by two coincided parallel lines. Once a contact is detected, constraints are imposed on the elements associated with the discontinuities. There are three possible contact states, i.e., open, closed and sliding, as shown in Figure 3. These three states may transfer dynamically under the coupled HM effects. In the original $\mathrm{NMM}$, no constraints are applied to the discontinuities in the open state. In this study, we assume fractures with touching of rough fracture surface and therefore have their own stiffness, satisfying Equation (7). Table 1 lists the constraints and their enforcement related to the three contact states.

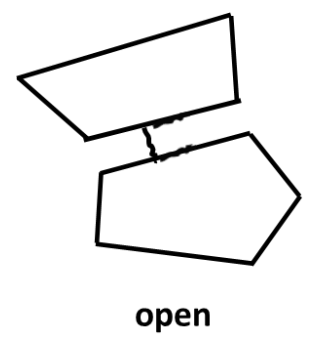

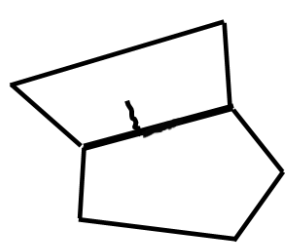

closed

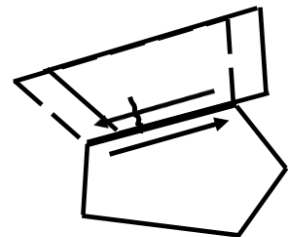

sliding

Figure 3 Three contact states: open, closed and sliding

Table 1 Contact constraints and their enforcement in three states

\begin{tabular}{l|ll}
\hline & \multicolumn{1}{|c}{ Constraints } & \multicolumn{1}{c}{ Constraints enforcement } \\
\hline Open & $\begin{array}{l}\text { Constitutive linear relationship in } \\
\text { normal and tangential directions }\end{array}$ & Developed in this study \\
Closed & \begin{tabular}{l} 
Displacement continuity in both \\
\cline { 2 - 2 }
\end{tabular} & Penalty method [20]
\end{tabular}




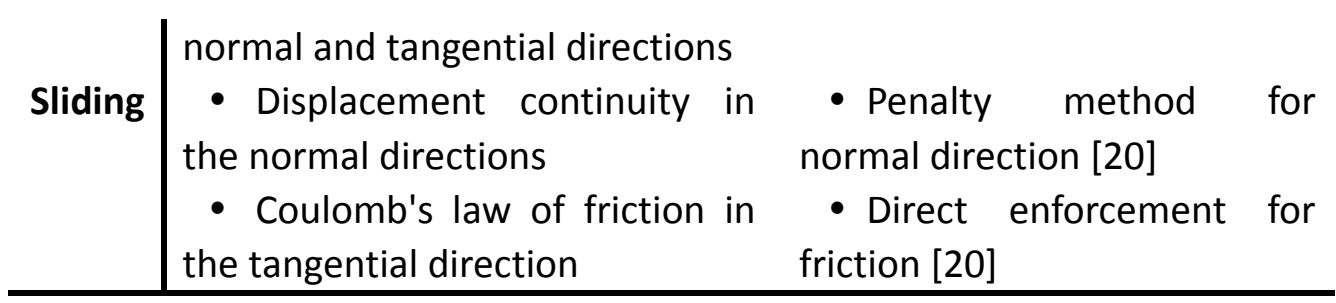

The work associated with closed and sliding states were derived by Shi [20]. For the closed state, continuity of displacement in both normal and tangential directions should be satisfied. This continuity is realized with penalty method, assuming high-stiffness springs are applied on the boundaries to avoid deviation of the continuity. The spring forces and displacements have linear relationship:

$$
\left(\begin{array}{c}
F_{n}^{\prime} \\
F_{s}^{\prime}
\end{array}\right)=\left(\begin{array}{ll}
g_{n} & 0 \\
0 & g_{s}
\end{array}\right)\left(\begin{array}{l}
d_{n} \\
d_{s}
\end{array}\right)
$$

where $F_{n}{ }^{\prime}$ and $F_{s}{ }^{\prime}$ are the hypothetic spring forces in the normal and tangential directions, $g_{n}$ and $g_{s}$ are the spring stiffness in these two directions with a very large number, $d_{n}$ and $d_{s}$ are the current-step distances between the contacted lines in these two directions. $d_{n}$ and $d_{s}$ are expressed by the previous-step coordinates and current-step displacements. Therefore, the potential energy associated with the enforcement of continuity constraint for the closed state is:

$$
\prod_{f c}=\frac{1}{2}\left(g_{n} d_{n}^{2}+g_{s} d_{s}^{2}\right)
$$

For sliding state, the continuity of displacement in the normal direction should be satisfied. In the tangential direction, the sliding surfaces satisfy Coulomb's law of friction. The frictional force is:

$$
F_{f}=F_{n}^{\prime} \tan \varphi \operatorname{sgn}\left(d_{s}\right)
$$

Where sgn() denotes the direction of sliding. Displacements of the two sliding surfaces are marked as $d_{s s}$ and $d_{s s}$, respectively. The corresponding potential energy associated with sliding state for the two surfaces are:

$$
\begin{aligned}
& \prod_{f s}=\frac{1}{2} g_{n} d_{n}{ }^{2}+g_{n} d_{n} \operatorname{sgn}\left(d_{s}\right) \tan \varphi d_{s s} \\
& \prod_{f s^{\prime}}=\frac{1}{2} g_{n} d_{n}{ }^{2}+g_{n} d_{n} \operatorname{sgn}\left(d_{s}\right) \tan \varphi d_{s s}{ }^{\prime}
\end{aligned}
$$

Now we derive the work for fractures in open state considering fractures stiffness in both normal and tangential directions. Assuming the stiffness constants, according to equation (7), we have:

$$
\left(\begin{array}{l}
d \sigma_{n}^{\prime} \\
d \sigma_{s}^{\prime}
\end{array}\right)=\left(\begin{array}{ll}
b_{m} k_{n} & 0 \\
0 & b_{m} k_{s}
\end{array}\right)\left(\begin{array}{l}
d \varepsilon_{n} \\
d \varepsilon_{s}
\end{array}\right)
$$

where $\varepsilon_{n}=\left(u_{n}^{r}-u_{n}^{r^{\prime}}\right) / b_{m}$ and $\varepsilon_{s}=\left(u_{s}^{r}-u_{s}^{r^{\prime}}\right) / b_{m}$. Then the strain energy stored in a fracture is 


$$
\Pi_{f o}=\int_{\Omega_{f}}\left(\sigma_{n}^{\prime} d \varepsilon_{n}+\sigma_{s}^{\prime} d \varepsilon_{s}\right) d \Omega=\frac{1}{2} \int_{\Gamma}\left[k_{n}\left(u_{n}^{r}-u_{n}^{r^{\prime}}\right)^{2}+k_{s}\left(u_{s}^{r}-u_{s}^{r^{\prime}}\right)^{2}\right] d s
$$

Combining with NMM approximation in Section 3, by minimizing the total potential energy we can derive the equilibrium equations.

Within each time step, open-close iteration is used to ensure the accurate detection and enforcement of the contact in the current step. Contact detection is conducted each time step and possibly leading to transferring among these three contact states and associated changes of constraint enforcement. These stringent routines enables the current model to genuinely model discrete fractures open, slip under coupled HM effects.

\subsection{A model for fluid flow in discrete fractures}

In 2D analysis, fluid flow in the fractures occurs in two directions: along the fractures, and flux exchange with the surrounding rock matrix in the direction normal to the fractures surfaces. To simulate such behavior we use a recently developed a practical model [29] for along-fracture and normal-to-fracture flow in the fracture network without introducing additional DOFs. Herein, we briefly introduce the basic idea of such a model [29] to deal with flow in discrete fractures.

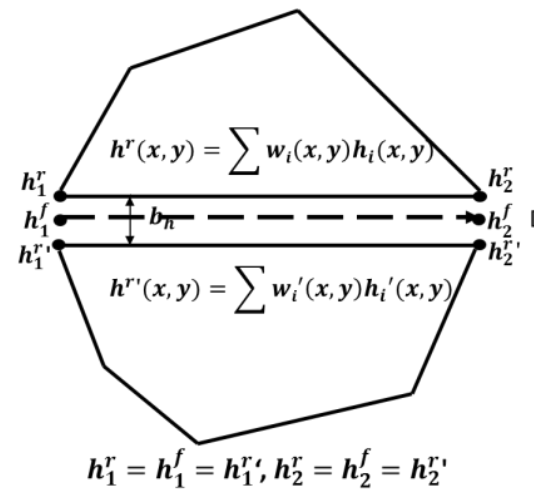

(a)

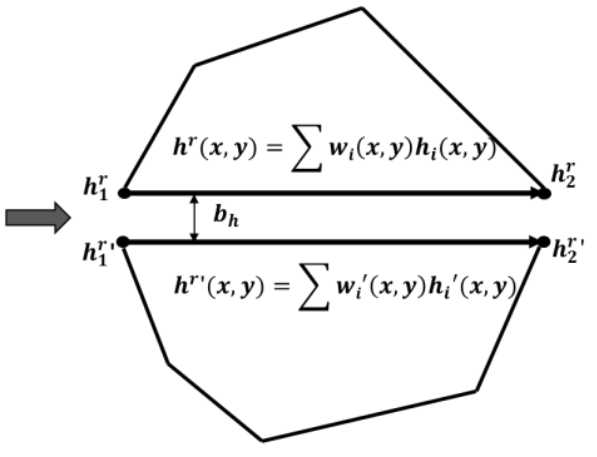

(b)

Figure 4 An approach for along-fracture flow without introducing DOFs for fractures

In the along-fracture direction, as shown in Figure 4a, fluid flow is driven by hydraulic head difference from $h_{1}^{f}$ to $h_{2}^{f}$. As the fracture is very thin, we assume hydraulic head within the fracture is uniform across its thickness. The fluid flow along the very thin fracture is represented by flow along its two surfaces satisfying the Darcy`s law. The two fracture surfaces belong to different elements. According to the energy-work seepage model that was developed in [26], the work that is associated with flow along a fracture is the summation of the work that is associated with flow along these two surfaces:

$$
W_{s}^{f}=\frac{1}{2} \gamma b_{h}\left(\int_{\Gamma} v_{s}^{f} \frac{\partial h^{r}}{\partial s} d s+\int_{\Gamma} v_{s}^{f} \frac{\partial h^{r^{\prime}}}{\partial s} d s\right)
$$

By using the local coordinate system and combining with Equation (22), we derive 
the work for along-fracture flow.

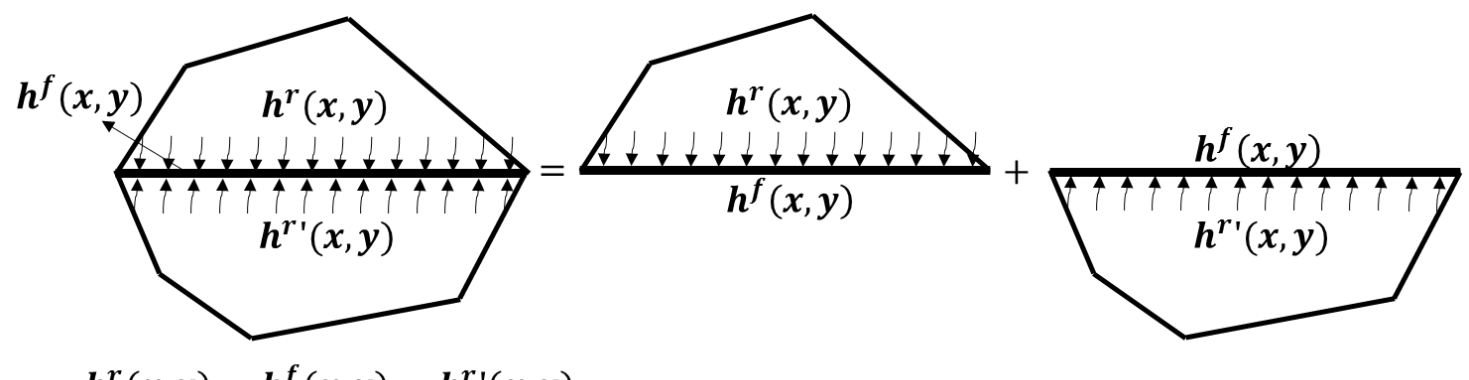

$h^{r}(x, y)=h^{f}(x, y)=h^{r '}(x, y)$

Figure 5 An approach for fracture-matrix flow without introducing DOFs for fractures

Similarly, the fracture-matrix flux exchange or the normal-to-fracture flow is modeled by considering flow across these two surfaces in their normal directions, as shown in Figure 5. Because the fractures are thin, the hydraulic head is assumed uniform in the direction normal to the fracture surfaces within the fractures. Therefore, a unique approach is provided by regarding those two surfaces as two Dirichlet boundaries, i.e., (1) one Dirichlet boundary with a given hydraulic head $h_{0}=h^{r}(x, y)$ for the fracture with an unknown hydraulic head $h^{f}(x, y)$ and (2) one Dirichlet boundary with a given hydraulic head $h_{0}=h^{r_{1}}(x, y)$ for the fracture with an unknown hydraulic head $h^{f}(x, y)$. With the assumption $h^{f}(x, y)=h^{r_{1}}(x, y)$ and $h^{f}(x, y)=$ $h^{r}(x, y)$, we can derive the work associated with fluid flow across each fracture surface. We further add them together and obtain the total work that is associated with normal-to-fracture flow on both surfaces of a fracture:

$$
W_{n}^{f}=-\gamma\left(\int_{\Gamma} k_{f n}\left[\frac{\partial h^{r^{\prime}}}{\partial n}\left(h^{r^{\prime}}-h^{r}\right)+\frac{\partial h^{r}}{\partial n}\left(h^{r}-h^{r^{\prime}}\right)\right] d s\right.
$$

As a rigorous model, fractures intersecting with Dirichlet and Neumann boundaries are also considered. For work associated with fractures intersecting with Dirichlet boundaries is:

$$
W_{D}^{f}=-\gamma \int_{\Gamma_{D}} k_{f}\left\{\frac{\partial h^{r}(x, y)}{\partial n_{D}}\left[h^{r}(x, y)-h_{0}(x, y)\right]+\frac{\partial h^{r^{\prime}}(x, y)}{\partial n_{D}}\left[h^{r^{\prime}}(x, y)-h_{0}(x, y)\right]\right\} d \Gamma
$$

Where $n_{D}$ is the normal direction of the Dirichlet boundary, $\Gamma_{D}$ is the fraction of the Dirichlet boundary in the fractures. For fractures intersecting with Neumann boundaries, the work is:

$$
W_{N}^{f}=\gamma\left(\int h^{r} \overline{q_{x}} d y+\int h^{r^{\prime}} \overline{q_{x}} d y+\int h^{r} \overline{q_{y}} d x+\int h^{r^{\prime}} \overline{q_{y}} d x\right)
$$

where $\left(\bar{q}_{x}, \bar{q}_{y}\right)$ are the known flux components.

\subsection{A fully coupled model for fractured rock}

By integrating the schemes in Sections 4.1, 4.2 and 4.3, we are able to construct a fully coupled system for complexly fractured and porous rock. Figure 6 shows the 
flowchart of the coupling routine with rigorous dynamic coupling of mechanical and fluid flow processes in the fracture-matrix system.

Note that though a fracture is mechanically closed, it may still conduct fluid through a residual hydraulic aperture, expressed by Equation (10). It is necessary to consider both along-fracture and normal-to-fracture fluid flow for all the mechanical states, i.e., open, closed, and sliding. When the mechanical aperture of a fracture is changing due to continuous opening or the change of contact state, its hydraulic aperture and hydraulic conductivity is updated. On the other hand, under the effect of deformation, fluid flow and their coupling, the contact state may dynamically change, and the corresponding constitutive behavior is described differently (see 4.2). Such a complex process may require a lot of open-close iterations to converge at each time step and achieve the accurate contact state and the corresponding enforcement in the global equations. Such a stringent scheme guarantees the accuracy of the results when considering complexly fractured rock and HM coupling is import. The above scheme realizes the indirect coupling. Thus, we are able to model coupled hydro-mechanical responses in complexly intersecting fractures.

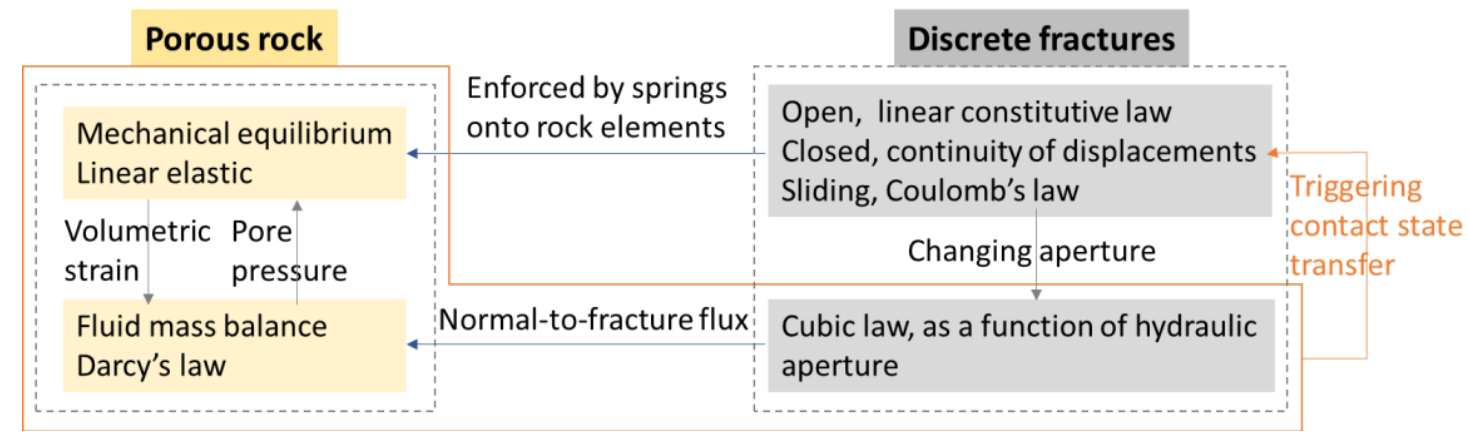

Figure 6 Flowchart of the fully coupled HM scheme for the fractured rock

\section{Examples and applications}

The model parts for direct coupling in porous media and for fluid flow in the discrete fractures were verified in the previous studies [29] and [32]. In this section we show some examples for coupled HM responses in the discrete fractured rock involving indirect coupling. We first verify the model by modeling coupled hydro-mechanical processes in rock with a single fracture involving one scenario with analytical solution. Then we simulate the case of rock with two sets of fractures with different scenarios. Last we demonstrate the capability of the new model to analyze coupled HM behavior in a complex fracture network induced by loading and injection.

\subsection{Example 1: HM coupling in porous rock with a single fracture}

In this example, we model a rock mass domain with a single fracture, with three different orientations. The geometrical and boundary conditions are as shown in Figure 7. The domains are $2 \times 2 \mathrm{~m}$ and we use same material properties for the three cases. The permeability contrast between porous rock and fractures is $1: 100,000$ initially. The Young 's modulus for rock matrix is $40 \mathrm{MPa}$, and the Poisson's ratio is 0.3 for Cases $a$ and $b$, and 0 for Case $c$. The residual hydraulic aperture of the fracture is 1 
$\mathrm{mm}$. We model two scenarios.

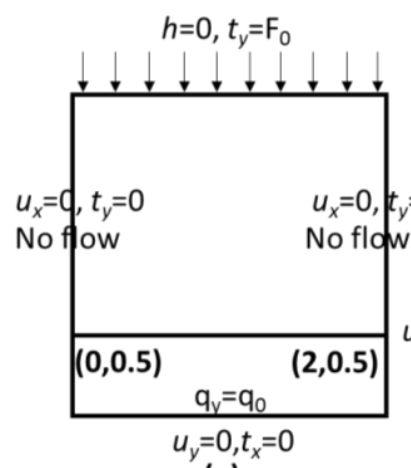

(a)

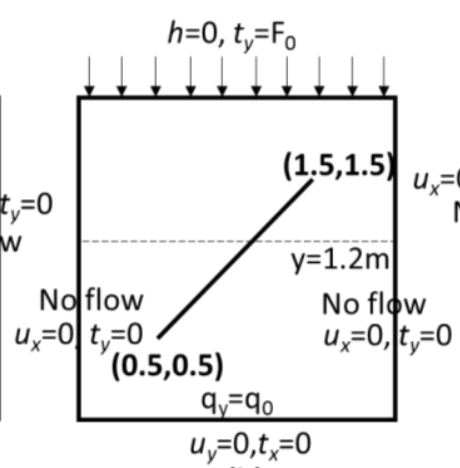

(b)

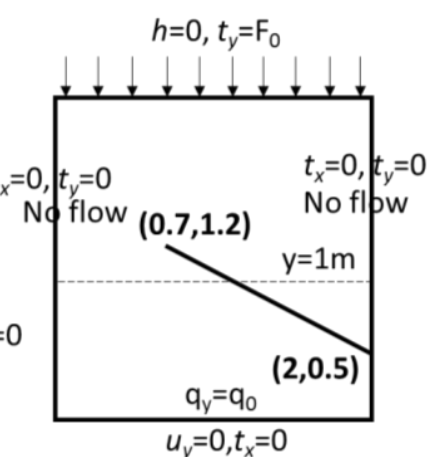

(c)

Figure 7 Geometrical and boundary conditions of the simulate examples

For Scenario 1, only fluid flow is considered. The flux $q_{0}$ at the bottom is $10^{-5} \mathrm{~m} / \mathrm{s}$. Figure 8 shows results for the three cases after reaching steady state. Theoretically for Case a, the hydraulic head distributes linearly in the vertical direction. From Figure $8 \mathrm{a}$ we see that the simulated result matches the theoretical solution, indicating good accuracy even with rather coarse mesh. For cases b and c, as the fractures are much more permeable than the rock matrix, we see that the hydraulic head is distorted but continuous across the fractures (Figures $8 \mathrm{~b}$ and $\mathrm{c}$ ).

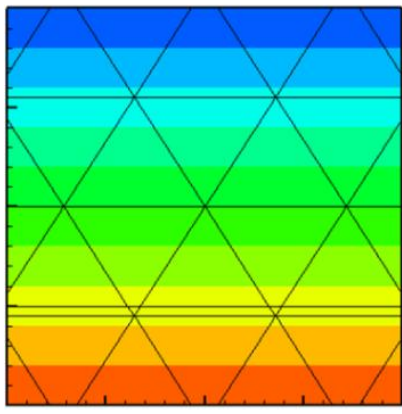

(a)

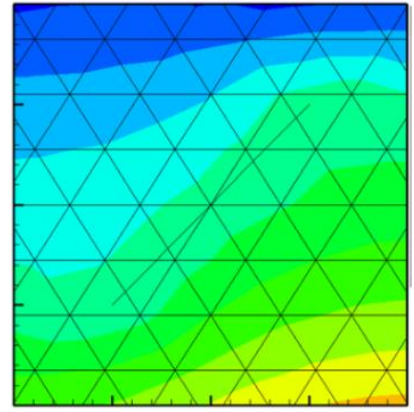

(b)

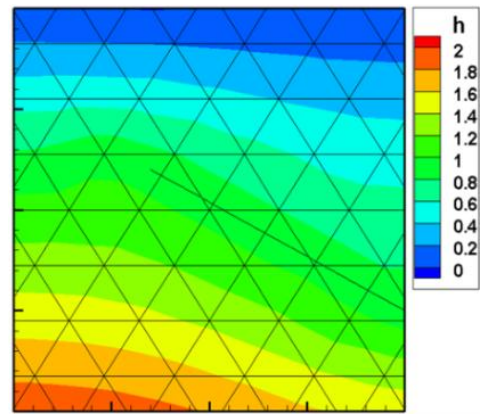

(c)

Figure 8 Hydraulic head (m) distribution with only fluid flow in Scenario 1

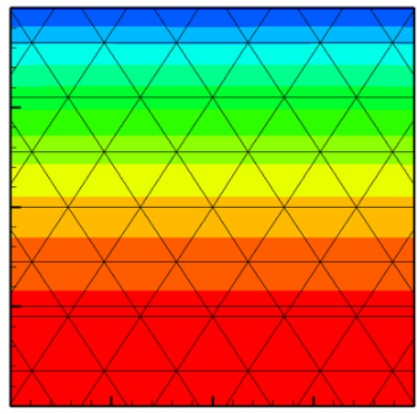

(a)

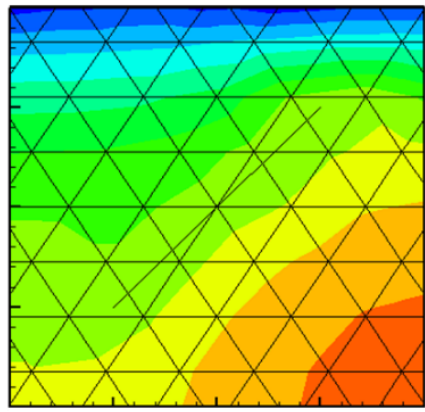

(b)

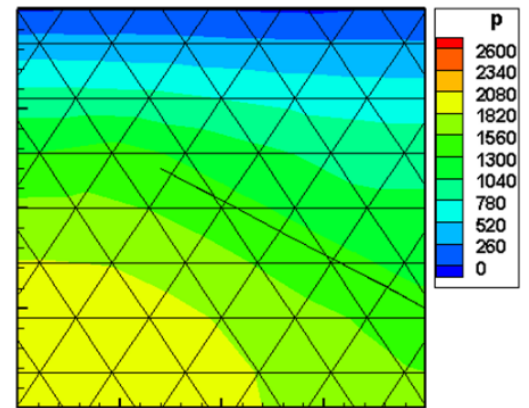

(c)

Figure 9 Hydraulic pressure $(\mathrm{Pa})$ distribution of coupled HM analysis after 100 seconds in Scenario 2

For Scenario 2, the bottom face is a non-flux boundary while a $10-\mathrm{kPa}$ loading is applied on the top face. Figure 9 shows the distribution of hydraulic head after 100 seconds, and Figure 10 shows the vertical displacement after 1200 seconds, when 
steady state has been reached. Figure 9 clearly shows the strong impact on the pressure distribution from the fractures and their orientations. The pressure change near the bottom of the models is the result of direct coupling between volumetric strain and pressure change. Such increase in fluid pressure will eventually drain towards the upper constant pressure head boundary. Figure 10 shows that the vertical displacement in case $c$ is much larger than those in cases $a$ and $b$, because no mechanical restriction is applied on the right face for case c.

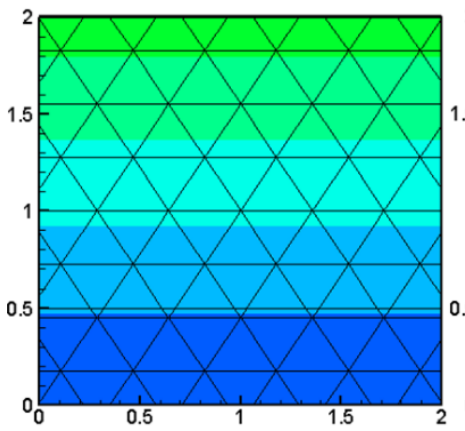

(a)

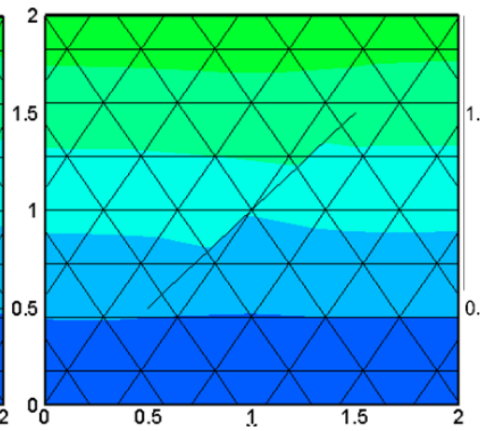

(b)

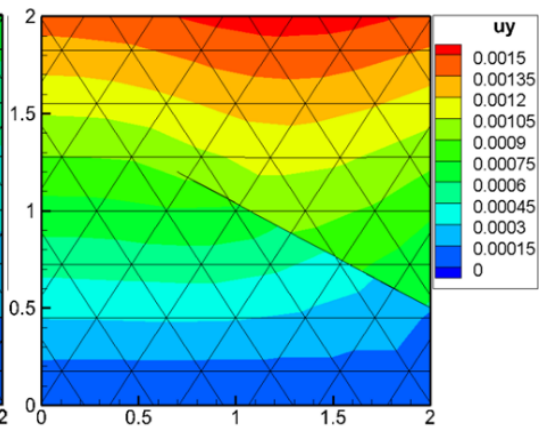

(c)

Figure 10 Vertical displacement (m) distribution of coupled HM analysis after 1200 seconds (i.e.: reaching steady sate) in Scenario 2

\subsection{Example 2: HM coupling in porous rock with two sets of fractures}

In this example, we calculate a porous rock domain with two sets of fractures, as shown in Figure 11. From an initial state with zero stress and zero fluid pressure, the model is subjected to loading on the top surface of $4 \mathrm{MPa}$. Fluid is injected at a constant pressure $0.2 \mathrm{MPa}$ from the end of a fracture intersecting the left boundary, and a fluid outlet at the right boundary-fracture intersection is maintained at $0 \mathrm{MPa}$. The properties of fractures and the rock matrix are listed in Table 2.

Table 2 Computation Parameters of example 2

\begin{tabular}{lll}
\hline Material & Parameter & Value \\
\hline Fluid & mass density, $\rho_{f}$ & $1000 \mathrm{~kg} / \mathrm{m}^{3}$ \\
& dynamic viscosity, $\mu_{f}$ & $1 \times 10^{-3} \mathrm{Ns} / \mathrm{m}^{2}$ \\
Rock Matrix & Young`s modulus & $4 \mathrm{GPa}$ \\
& Poisson's ratio & 0.3 \\
& permeability coefficient & $1 \times 10^{-13} \mathrm{~m} / \mathrm{s}$ \\
& Biot-Willis coefficient, $\alpha$ & 1 \\
& Biot's modulus, $\mathrm{M}$ & $\infty$ \\
\multirow{5}{*}{ Fractures } & mass density & $2300 \mathrm{~kg} / \mathrm{m}^{3}$ \\
& initial mechanical aperture & $0 \mathrm{~m}$ \\
& shear stiffness & $10^{10} \mathrm{~Pa} / \mathrm{m}$ \\
& normal stiffness & $10^{10} \mathrm{~Pa} / \mathrm{m}$ \\
& factor, $f$ & 1 \\
& residual hydraulic aperture, $b_{h r}$ & $10 \mu \mathrm{m}$ \\
& Friction angle & 0 \\
\hline
\end{tabular}




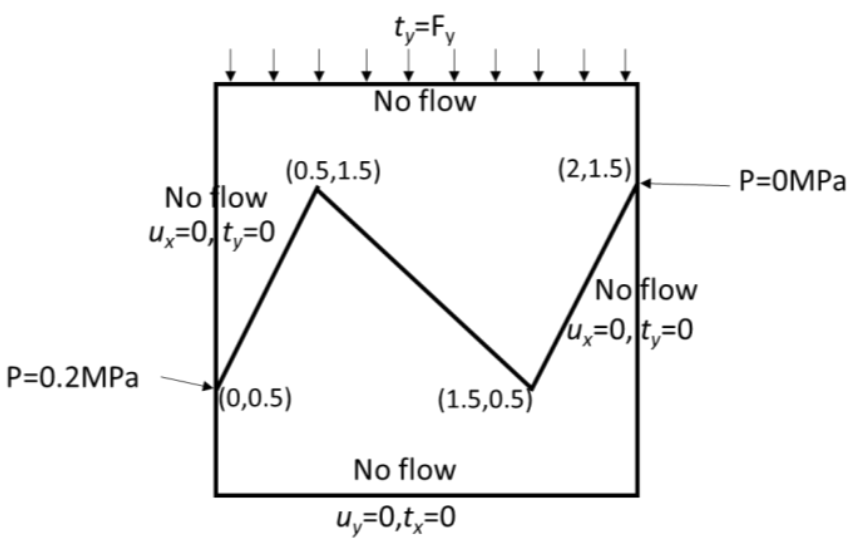

Figure 11 Geometrical and boundary condition of the simulate examples

We first run simulations without fluid flow and mechanics coupling. In this case, both fluid flow and mechanical analysis are under steady state. Figure 12 shows the distribution of hydraulic head, horizontal and vertical displacements. As the Dirichlet boundary condition is only applied at the fracture ends, hydraulic head concentrates at the two ends (see Figure 12a). The horizontal and vertical displacements shown in Figure $12 \mathrm{~b}$ and $\mathrm{c}$ are results of the applied loading on the upper boundary, indicating the discontinuous displacement field across the deformable fractures.

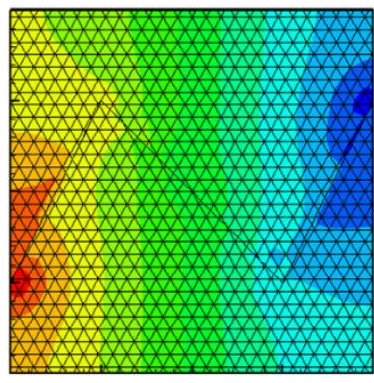

(a)
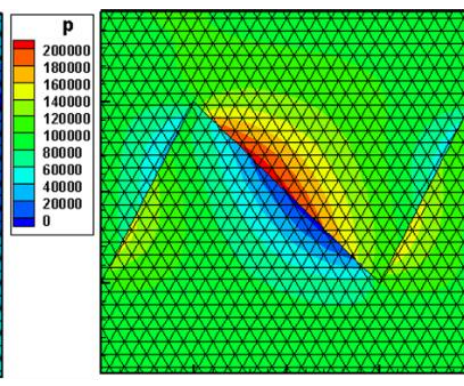

(b)

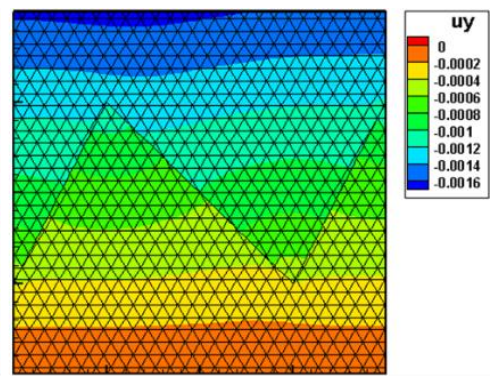

(c)

Figure 12 Simulated results without coupling: (a) pressure $(\mathrm{Pa}),(\mathrm{b})$ horizontal displacement $(\mathrm{m})$ and $(\mathrm{c})$ vertical displacement $(\mathrm{m})$

Then we activate the coupled hydro-mechanical analysis. Figure 13 shows the evolution of fluid pressure. We clearly see that the fractures, as dominant conduit, have a great impact on the fluid distribution over the entire field. As a result of the stress increase, fluid pressure goes up under poro-elastic effects, especially in the low permeability rock matrix. However, this excess fluid pressure is drained into the conductive fractures eventually. Figures 14 and 15 shows the evolution of horizontal and vertical displacements. The horizontal displacements in Figure 14 are restricted by the lateral no displacement boundaries, but the very early transient response shows that the rock matrix can expand into the sub-vertical softer fractures as long as the pressure in the fractures are low (see in Figure 14, 1 day, red and blue displacement contours adjacent to two fractures). The vertical displacements shown in Figure 15 are dominant downwards due to the force applied on the top boundary and the discontinuous displacement across fractures are very apparent. 


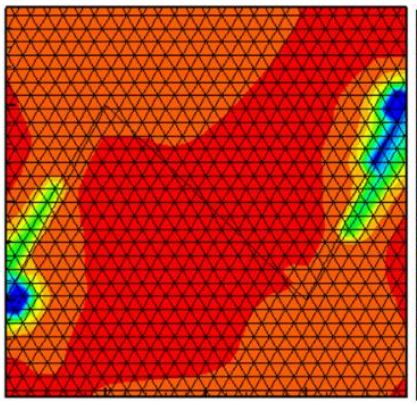

$t=1 d$

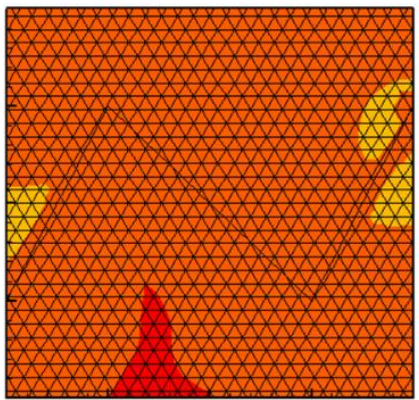

$t=5 d$

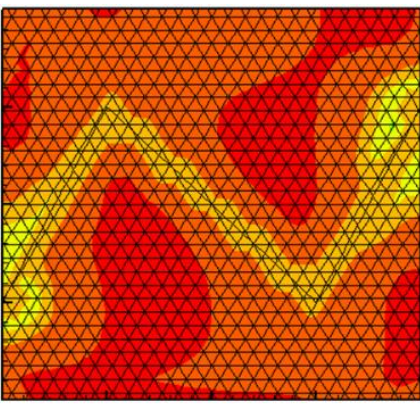

$\mathbf{t}=\mathbf{2 d}$

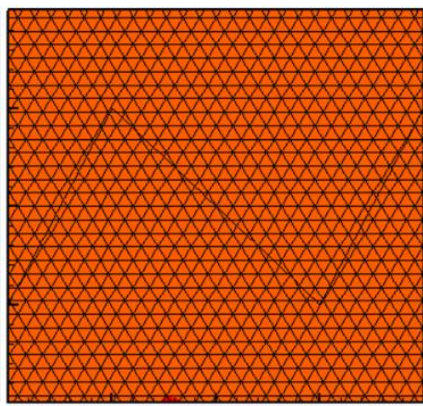

$t=8 d$

Figure 13 Simulated evolution of fluid pressure $(\mathrm{Pa})$

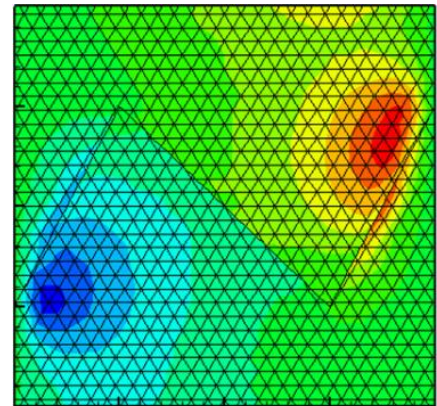

$t=1 d$

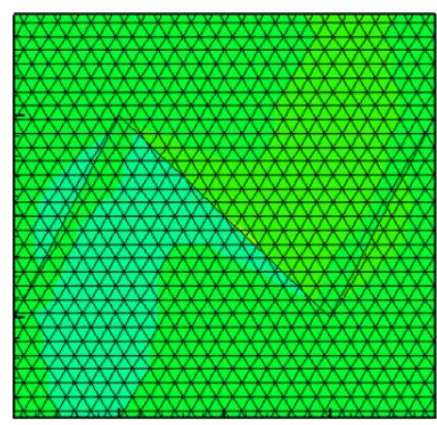

$t=5 d$

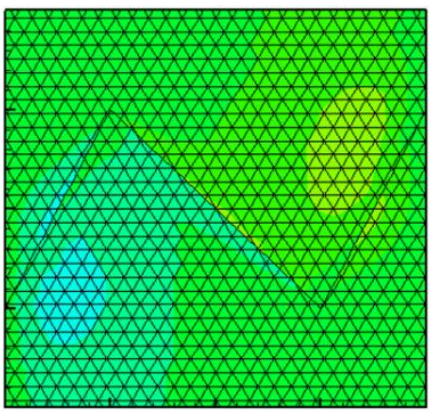

$t=2 d$

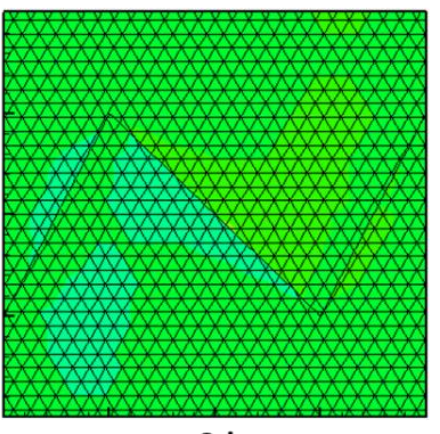

$t=8 d$

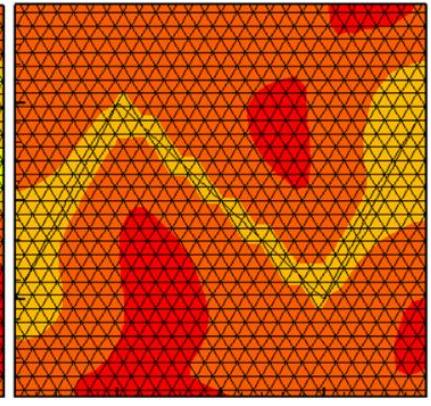

$t=3 d$

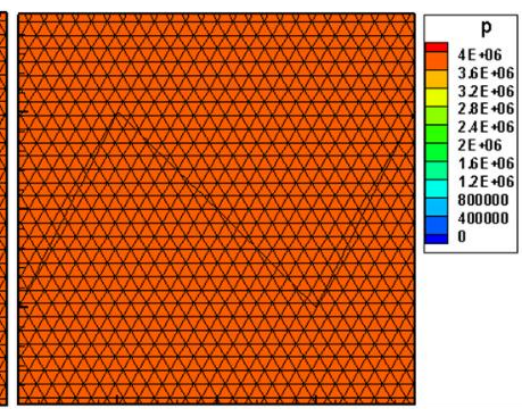

$t=10 d$

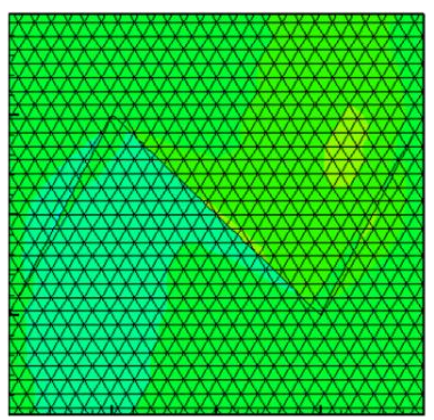

$t=3 d$

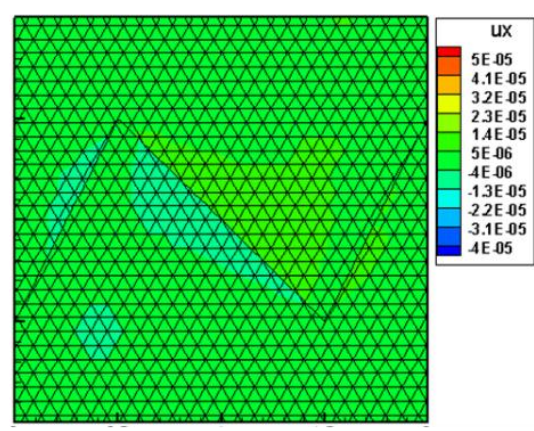

$\mathrm{t}=\mathbf{1 0 d}$

Figure 14 Simulated evolution of horizontal displacement $(\mathrm{m})$ 


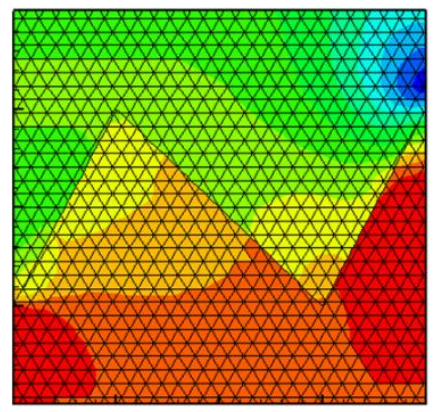

$\mathbf{t}=\mathbf{1 d}$

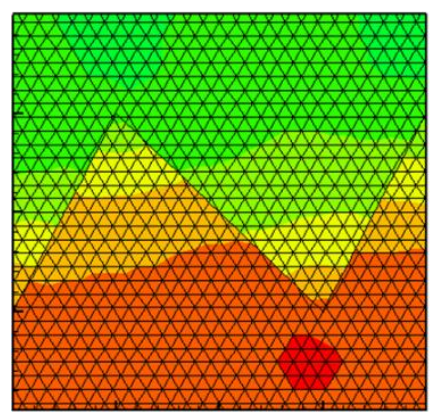

$t=5 d$

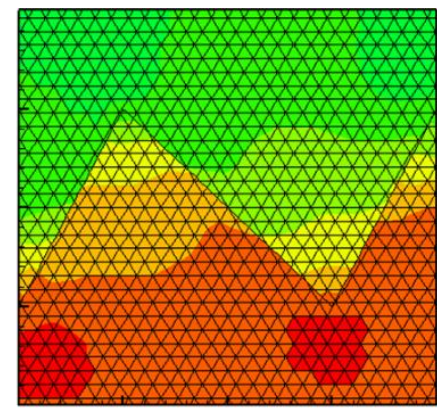

$t=2 d$

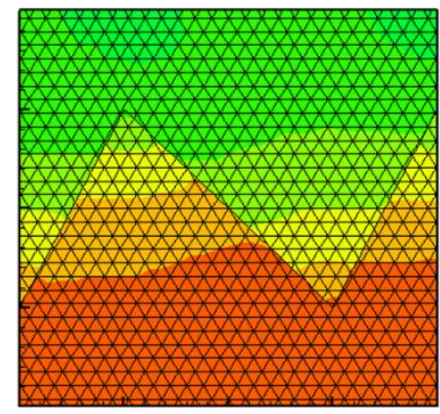

$t=8 d$

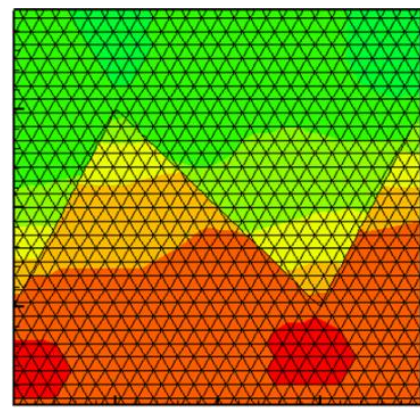

$t=3 d$

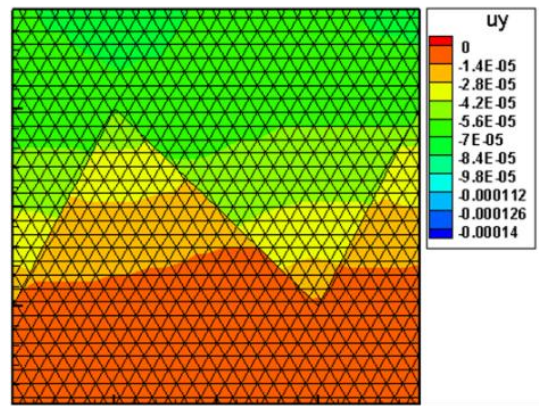

$t=10 d$

Figure 15 Simulated evolution of vertical displacement $(\mathrm{m})$

5.3 Applications: coupled HM processes in porous rock involving a complex fracture network

In the following we present a number of simulation examples with the purpose of testing and demonstrating the modeling of key coupled HM processes for various types of applications. We consider a complex fracture network involving 25 fractures of various orientations and lengths with multiple intersections embedded in a porous rock matrix. For each of the simulation examples we apply the same fracture geometry but vary the scale of the problem domain and fracture network. These demonstration examples are not intended to be completely realistic related to each application, but is intended for demonstration of the models capability of capturing key coupled processes in each type of application. The applications involve (1) mechanical compaction of a fractured rock mass, and (2) fluid injection from a borehole.

\subsubsection{Mechanical compaction of a porous and fractured rock mass}

In this application, we first analyze mechanical compaction of a $10 \times 10 \mathrm{~m}$ rock mass domain. This application is designed to represent coupled HM processes that may occur when a shallow rock foundation is subjected to mechanical loading from a surface structure. The rock mass domain is subject to an instantaneous loading in the vertical and horizontal direction by applying tractions of $6 \mathrm{MPa}$ on the left and bottom surfaces. Dirichlet (constant head) boundaries are assumed the left and right faces, with hydraulic heads of $10 \mathrm{~m}$ and $0 \mathrm{~m}$, respectively. The properties of fractures and the rock matrix are listed in Table 3. 


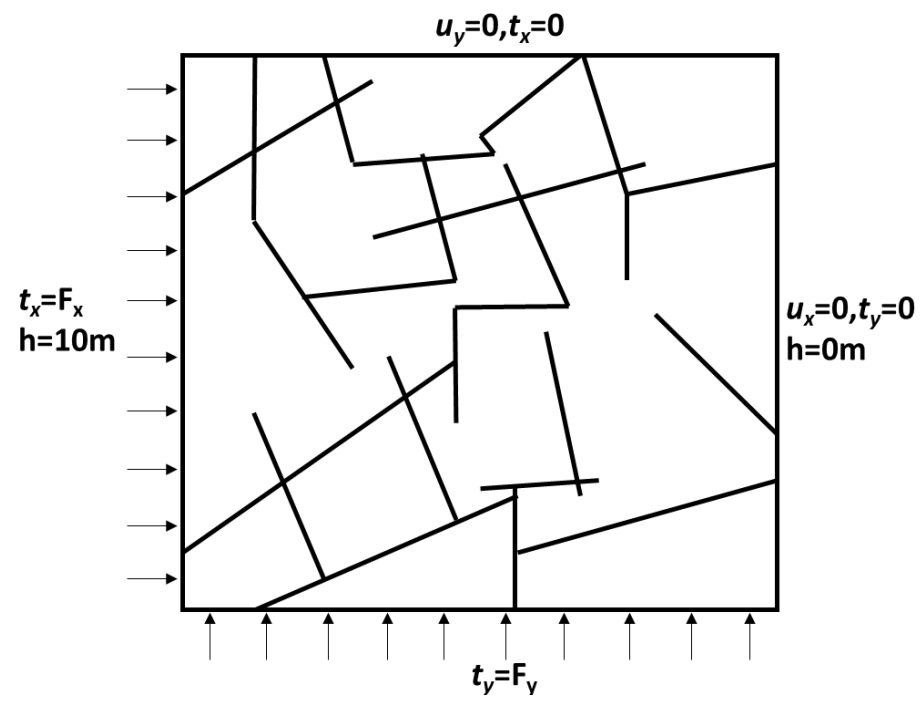

Figure 16 Geometrical and boundary condition of the simulate examples

Table 3 Computation Parameters of application

\begin{tabular}{lll}
\hline Material & Parameter & Value \\
\hline Fluid & mass density, $\rho_{f}$ & $1000 \mathrm{~kg} / \mathrm{m}^{3}$ \\
& dynamic viscosity, $\mu_{f}$ & $1 \times 10^{-3} \mathrm{Ns} / \mathrm{m}^{2}$ \\
Rock Matrix & young's modulus & $40 \mathrm{GPa}$ \\
& Poisson's ratio & 0.3 \\
& permeability coefficient & $1 \times 10^{-13} \mathrm{~m} / \mathrm{s}$ \\
& Biot-Willis coefficient, $\alpha$ & 1 \\
& Biot's modulus, $M$ & $\infty$ \\
Fractures & mass density & $2300 \mathrm{~kg} / \mathrm{m}^{3}$ \\
& initial mechanical aperture & $0 \mathrm{~m}$ \\
& shear stiffness & $4 \times 10^{10} \mathrm{~Pa} / \mathrm{m}$ \\
& normal stiffness & $4 \times 10^{10} \mathrm{~Pa} / \mathrm{m}$ \\
& factor, $f$ & 1 \\
& residual hydraulic aperture, $b_{h r}$ & $10 \mu \mathrm{m}$ \\
& Friction angle & 0 \\
\hline
\end{tabular}

Figure 17 shows the evolution of fluid pressure. First, there is a poro-elastic increase of fluid pressure as a result of compaction when adding the $6 \mathrm{MPa}$ tractions at the bottom and left surfaces. First the pressure instantaneously in matrix goes up approximately $6 \mathrm{MPa}$ in the rock matrix, though some variations occurs as a results of heterogeneous stress and strain changes in the model. Thereafter, the pressure in the matrix is gradually decreasing by fluid drainage into the connected fracture network and by fluid flowing out through the left and right boundaries that are set at constant heads. Figures 18 and 19 show the evolution of horizontal and vertical displacements. From the figures we can see that after 600 days the whole system almost reaches steady state while some significant transient effects occur in the early time. The horizontal displacements shown first respond to mechanical compaction that are somewhat affected by the lower fractures (Figure 18a). The most substantial 
horizontal compaction occurs as a results of poro-elastic shrinkage of the rock matrix when drained during the first 100 days (Figures 18b and c). Similarly, the vertical displacement shows that most compaction occurs during the first 100 days as a results of drainage of the rock matrix. This application demonstrates the capability of the new model for analyzing coupled HM processes related to compaction and drainage in porous rock with complexly intersected fractures.

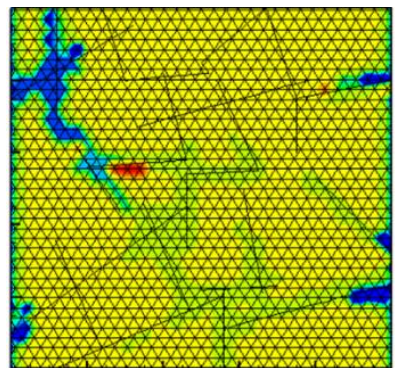

$t=1 d$

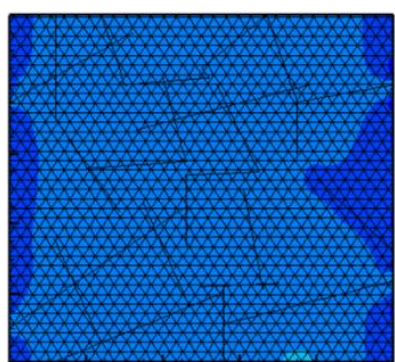

$t=200 d$

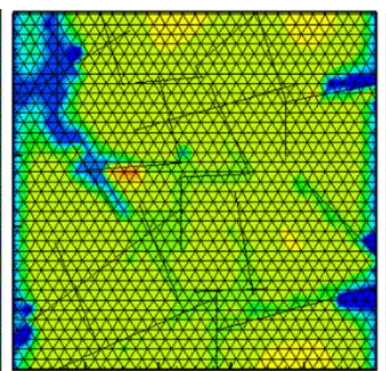

$\mathrm{t}=10 \mathrm{~d}$

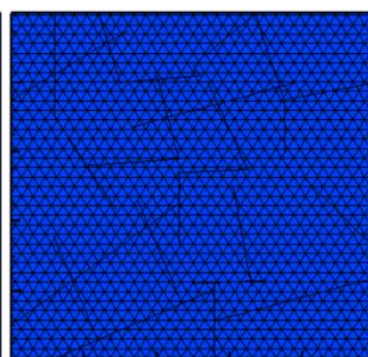

$t=300 d$

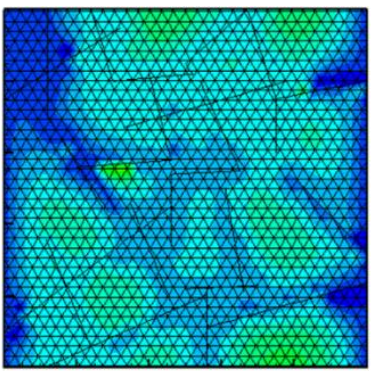

$t=100 d$

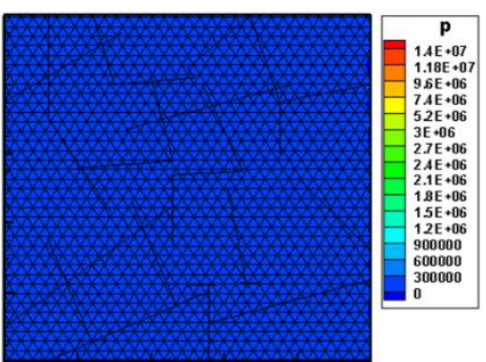

$t=600 d$

Figure 17 Simulated evolution of fluid pressure $(\mathrm{Pa})$

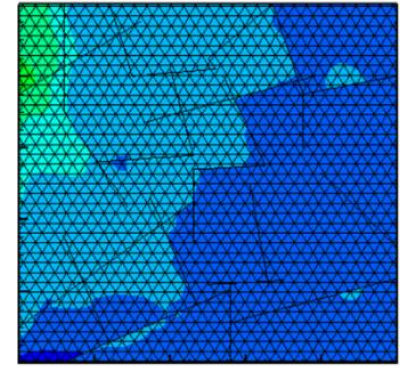

$t=1 d$

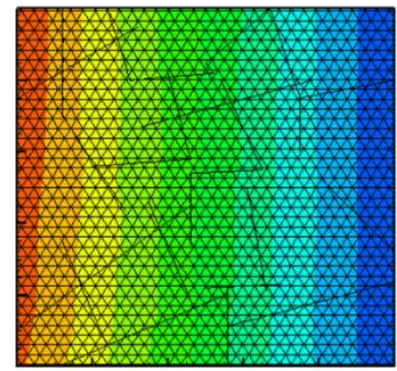

$t=200 d$

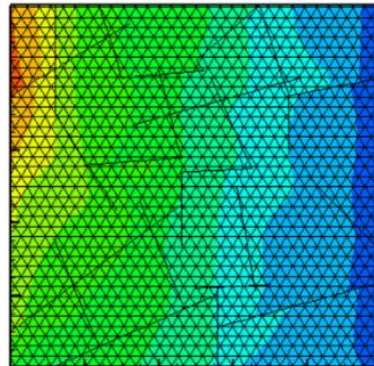

$t=10 d$

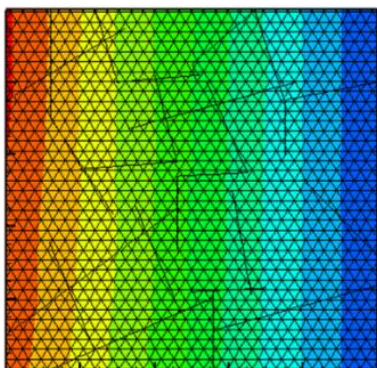

$t=300 d$

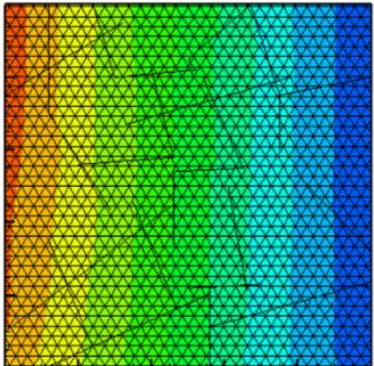

$t=100 d$

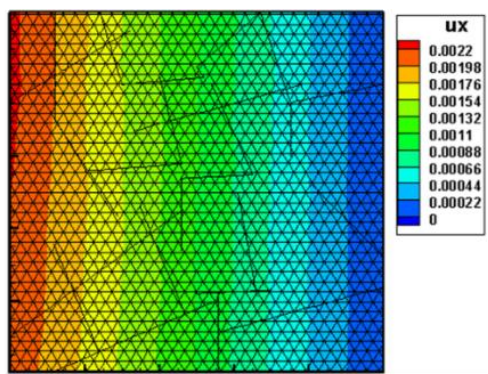

$t=600 d$

Figure 18 Simulated evolution of horizontal displacement (m) 


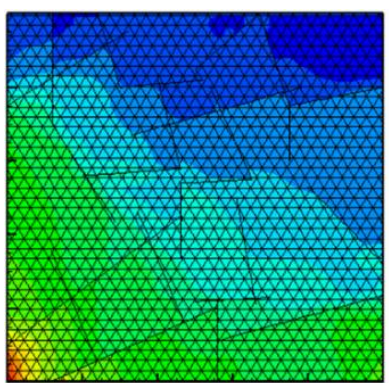

$t=1 d$

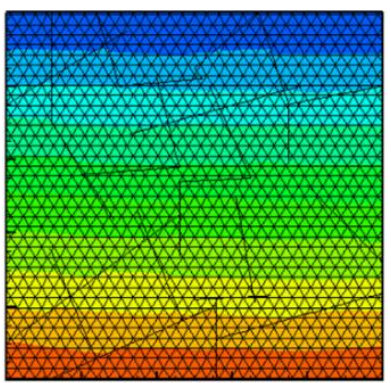

$\mathrm{t}=200 \mathrm{~d}$

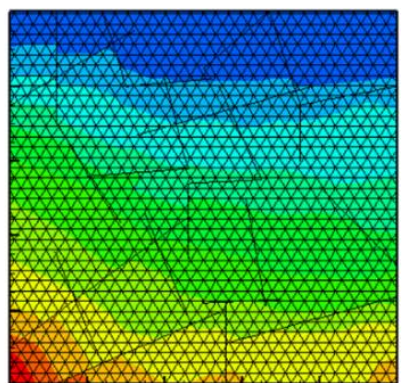

$t=10 d$

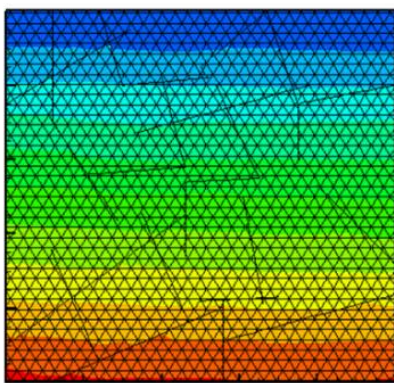

$t=300 d$

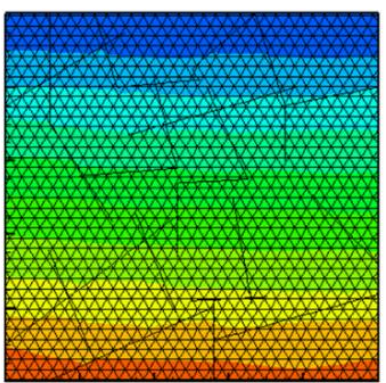

$t=100 d$

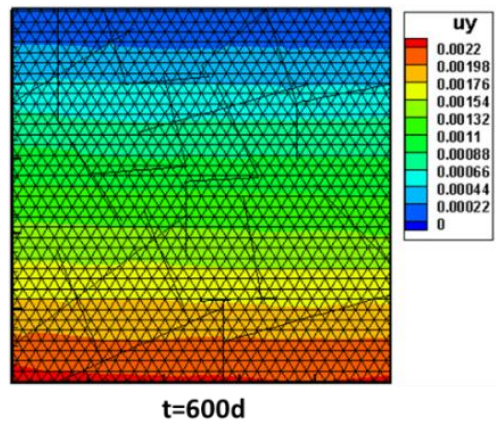

Figure 19 Simulated evolution of vertical displacement (m)

We further apply the model to a rather soft $100 \times 100 \mathrm{~m}$ porous domain, with the same geometry of distributed fractures. The domain is subjected to a $10 \mathrm{kPa}$ traction on the top surface with a hydraulic head of $0 \mathrm{~m}$. The other three boundaries are impermeable. And the initial hydraulic head is $h=100 \mathrm{~m}$ within the entire domain. The properties of the fractures and the rock matrix are listed in Table 4.

Table 4 Computation Parameters

\begin{tabular}{lll}
\hline Material & Parameter & Value \\
\hline Fluid & mass density, $\rho_{f}$ & $1000 \mathrm{~kg} / \mathrm{m}^{3}$ \\
Rock Matrix & dynamic viscosity, $\mu_{f}$ & $1 \times 10^{-3} \mathrm{Ns} / \mathrm{m}^{2}$ \\
& young's modulus & $4 \mathrm{MPa}$ \\
& Poisson's ratio & 0 \\
& permeability coefficient & $2.5 \times 10^{-8} \mathrm{~m} / \mathrm{s}$ \\
& Biot-Willis coefficient, $\alpha$ & 1 \\
& Biot's modulus, $\mathrm{M}$ & $\infty$ \\
& mass density & $2300 \mathrm{~kg} / \mathrm{m}^{3}$ \\
& initial mechanical aperture & $0 \mathrm{~m}$ \\
& shear stiffness & $1 \times 10^{6} \mathrm{~Pa} / \mathrm{m}$ \\
& normal stiffness & $1 \times 10^{6} \mathrm{~Pa} / \mathrm{m}$ \\
& factor, $f$ & 1 \\
& residual hydraulic aperture, $b_{h r}$ & $10 \mu \mathrm{m}$ \\
& Friction angle & 0 \\
\hline
\end{tabular}




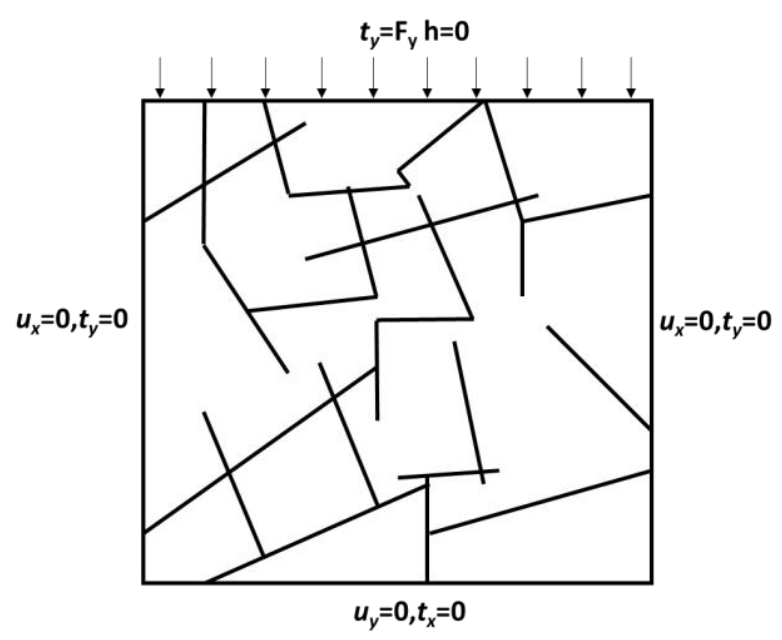

Figure 20 Geometrical and boundary condition of the simulate examples

Figure 21 shows the evolution of fluid pressure. First, there is a poro-elastic increase of fluid pressure as a result of compaction after adding the $10 \mathrm{kPa}$ traction at the top surface. Thereafter, the pressure in the matrix is gradually decreasing by fluid drainage into the connected fracture network system and by fluid flowing out through the top boundary. As the initial permeability contrast between fracture and porous media is not very high, we don 't see very obvious fracture-dominant drainage for this problem. As the rock and fractures are not very stiff, we see obvious fracture opening after 600 days, as shown in the deformed mesh in Figures 21 and 22. Figures 22 and 23 show the evolution of horizontal and vertical displacements. From these figures we can see that after 1000 days, the whole system almost reaches steady state. Specifically, the horizontal displacement contour (Figure 22b) shows rough distribution of the opened fractures after 1000 days. This application demonstrates an extreme case of loading and drainage of a saturated fractured media that may lead to long-term large ground surface subsidence.

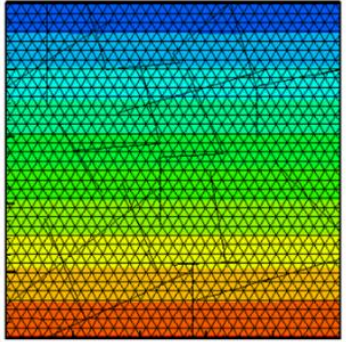

$t=100$ days

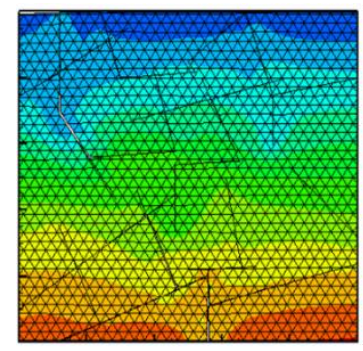

$t=600$ days

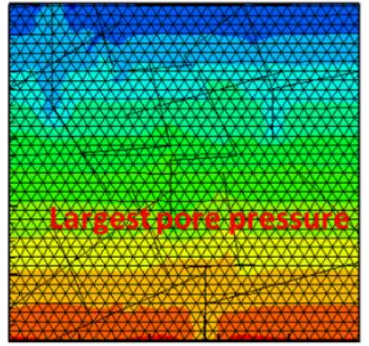

$\mathbf{t}=\mathbf{2 0 0}$ days

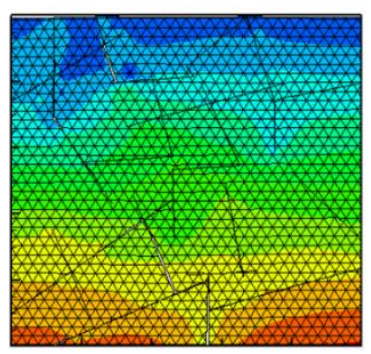

$\mathrm{t}=\mathbf{8 0 0}$ days

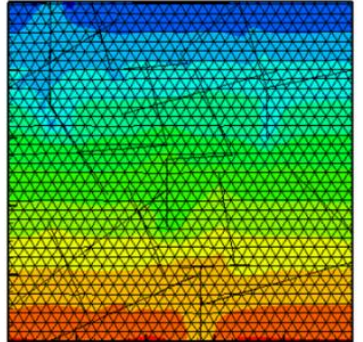

$\mathbf{t}=\mathbf{3 0 0}$ days

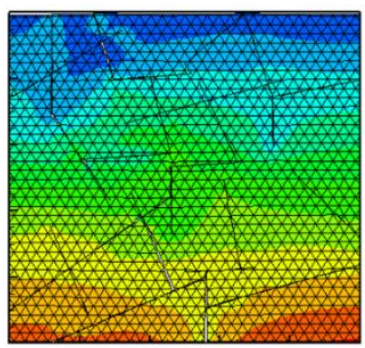

$t=1000$ days

Figure 21 Simulated evolution of fluid pressure $(\mathrm{Pa})$ 


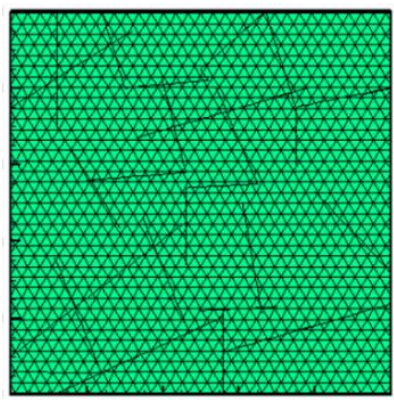

$\mathbf{t}=\mathbf{1 0 0}$ days

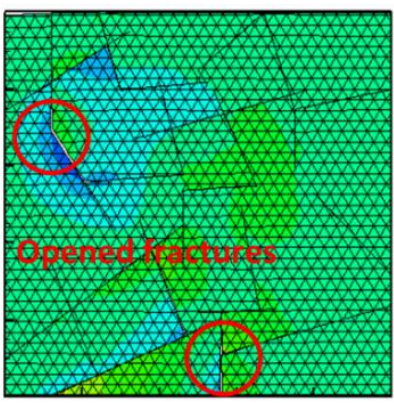

$t=600$ days

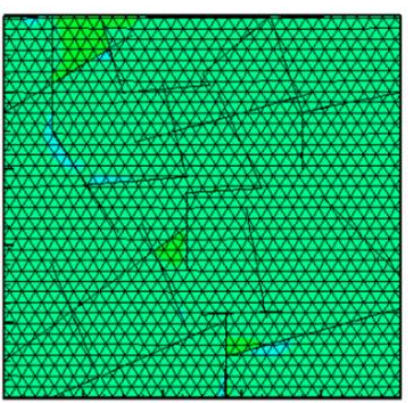

$\mathbf{t}=\mathbf{2 0 0}$ days

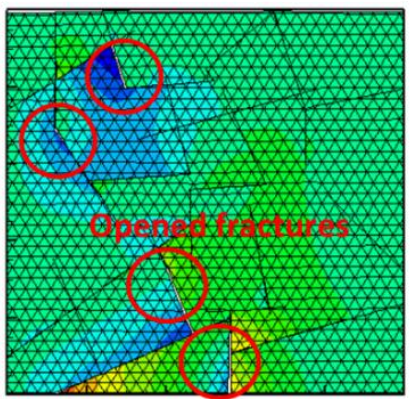

$t=800$ days

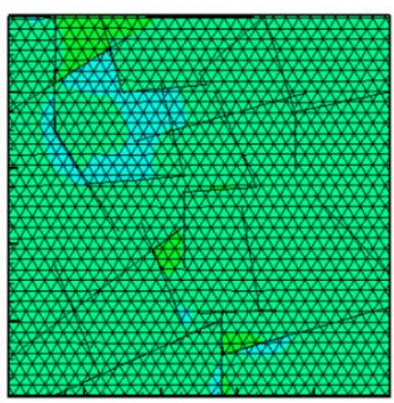

$t=300$ days

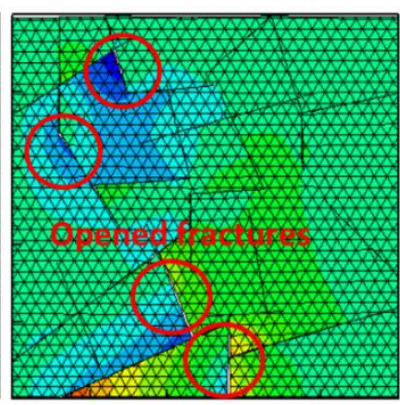

$t=1000$ days

(a)

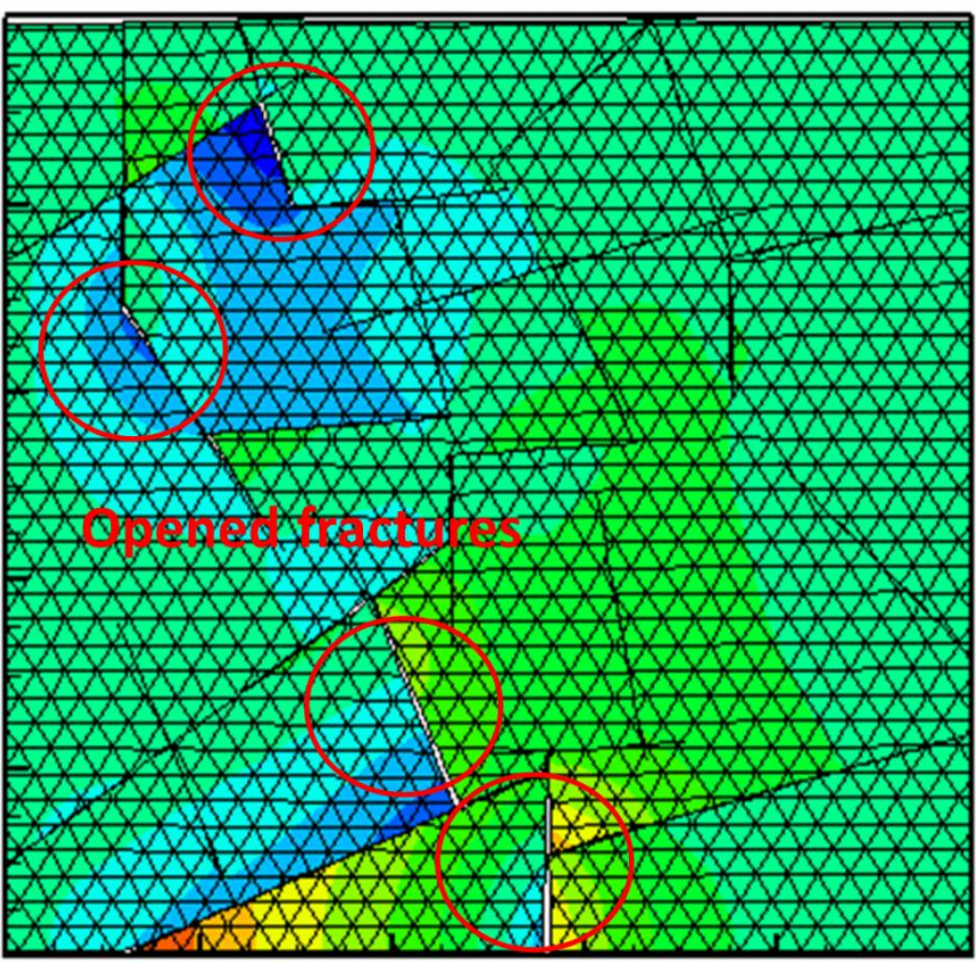

(b)

Figure 22 (a) Simulated evolution of horizontal displacement (m), (b) enlarged contour of horizontal displacement $(\mathrm{m})$ for $\mathrm{t}=1000$ days 


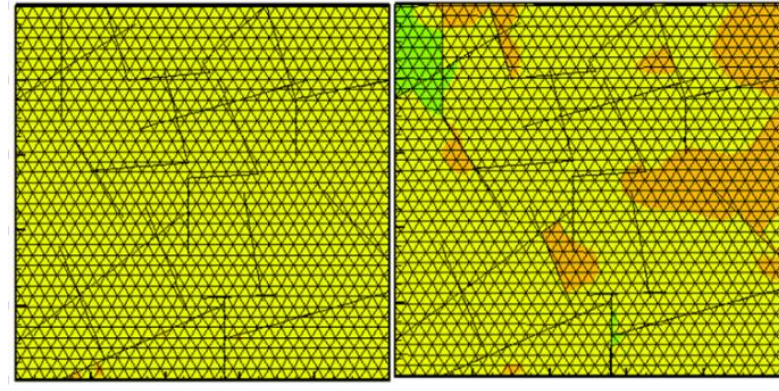

$\mathbf{t}=100$ days

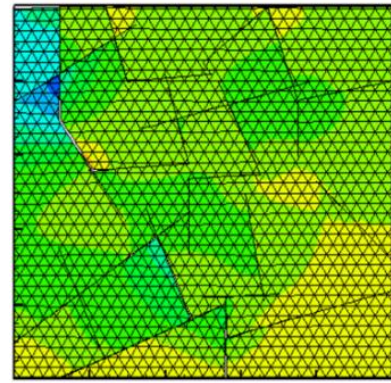

$t=600$ days $t=200$ days

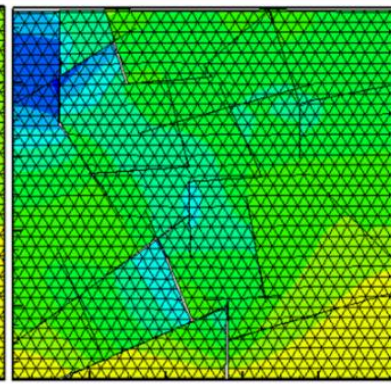

$\mathbf{t}=\mathbf{8 0 0}$ days

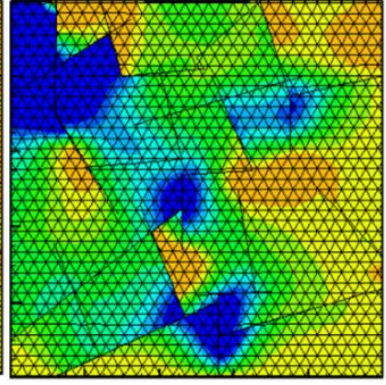

$t=300$ days

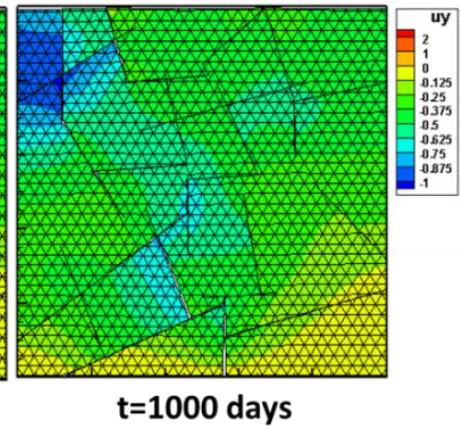

Figure 23 Simulated evolution of vertical displacement $(\mathrm{m})$

\subsubsection{Fluid injection from a borehole}

We simulate fluid injection from a vertical borehole (diameter $0.1 \mathrm{~m}$ ) at the center of a fractured model domain now scaled to size of $1 \times 1 \mathrm{~m}$ (Figure 24). This example represent near borehole coupled $\mathrm{HM}$ effects that takes place during injection. The initial total stress is assumed to be $35 \mathrm{MPa}$ and $30 \mathrm{MPa}$, in the two horizontal and directions, respectively, whereas the initial fluid pressure is $5 \mathrm{MPa}$. All boundaries are fixed with fixed pressure head. The water is injected at changing pressure from the borehole, as shown in Figure 25. We calculate the domain HM response under injection. The parameters of fractures and the rock matrix are listed in Table 5.

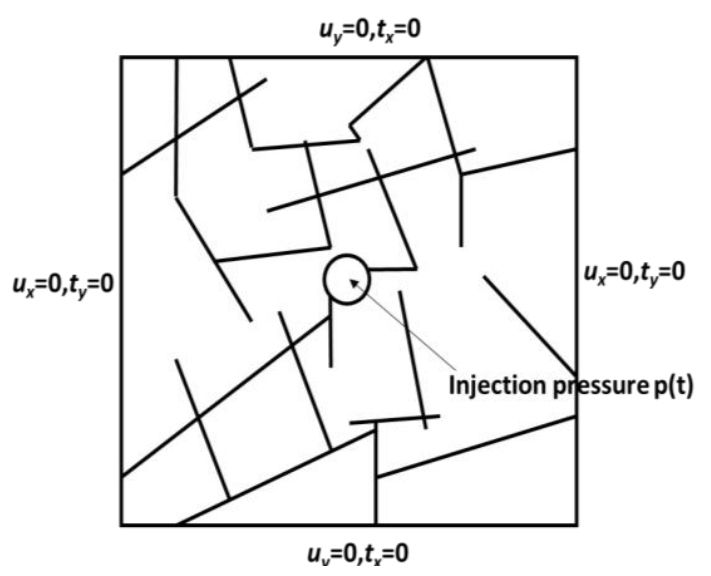

Figure 24 Geometrical and boundary condition of the simulate examples 


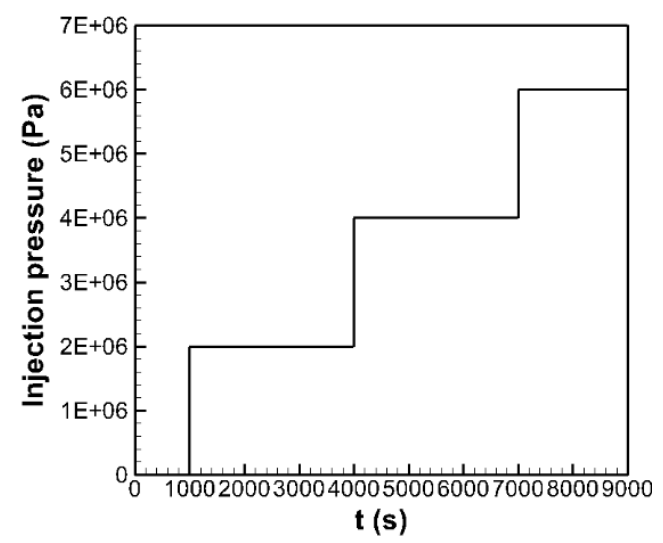

Figure 25 Injected pressure at the wellbore

Table 5 Computation Parameters of application

\begin{tabular}{lll}
\hline Material & Parameter & Value \\
\hline Fluid & mass density, $\rho_{f}$ & $1000 \mathrm{~kg} / \mathrm{m}^{3}$ \\
& dynamic viscosity, $\mu_{f}$ & $1 \times 10^{-3} \mathrm{Ns} / \mathrm{m}^{2}$ \\
Rock Matrix & young's modulus & $10 \mathrm{GPa}$ \\
& Poisson's ratio & 0.3 \\
& permeability coefficient & $1 \times 10^{-10} \mathrm{~m} / \mathrm{s}$ \\
& Biot-Willis coefficient, $\alpha$ & 1 \\
& Biot's modulus, M & $10 \mathrm{GPa}$ \\
& mass density & $2000 \mathrm{~kg} / \mathrm{m}^{3}$ \\
& initial mechanical aperture & $0 \mathrm{~m}$ \\
& shear stiffness & $1 \times 10^{10} \mathrm{~Pa} / \mathrm{m}$ \\
& normal stiffness & $1 \times 10^{10} \mathrm{~Pa} / \mathrm{m}$ \\
& factor, $f$ & 1 \\
& residual hydraulic aperture, $b_{h r}$ & $10 \mu \mathrm{m}$ \\
& Friction angle & 0 \\
\hline
\end{tabular}

Figure 26 shows the evolution of the injection-induced fluid pressure. At 1000 seconds, highest pressure changes occurs in the matrix adjacent to the well (Figure 26 upper right). Although fluid pressure at this early stage also penetrates into fractures it is not enough to build up a substantial pressure within the fracture network. At 4000 seconds, the well pressure has been elevated by $2 \mathrm{MPa}$ for 3000 seconds (Figure 25) and substantial pressure increase can be observed in the fracture network (Figure 26). At 4000 seconds some of the pressure increase with the matrix has been released by opening of fractures and associated fluid flow to outer constant pressure boundaries. This behavior is repeated with each pressure step and finally at 9000 seconds, the increased pressure occupy a large part of the domain because of relatively high fracture apertures around the well. The fracture network responses are in this case complex as it involves primary opening of fractures due to pressure increase within the fractures, but also pore-elastic expansion of rock matrix that tends to expand into the softer fractures and close them. Thus, this example demonstrates the capability to model coupled hydro-mechanical processes including 
both direct coupling (such as poro-elastic expansion of matrix) and indirect coupling (such as permeability change in fractures) involving a complex fracture network with multiple intersections.

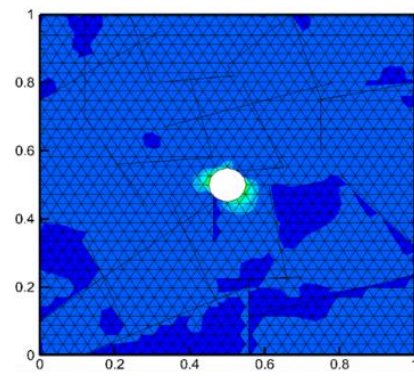

$1000 \mathrm{~s}$

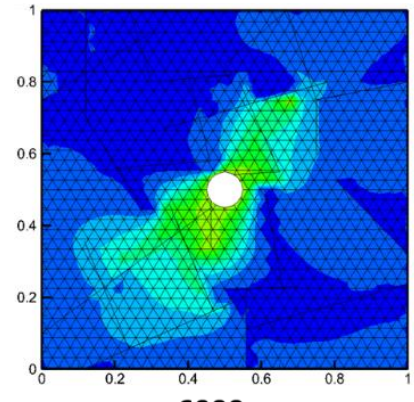

$6000 \mathrm{~s}$

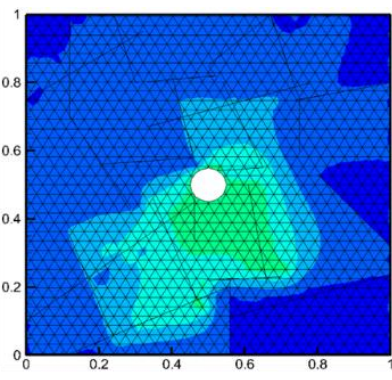

$3000 \mathrm{~s}$

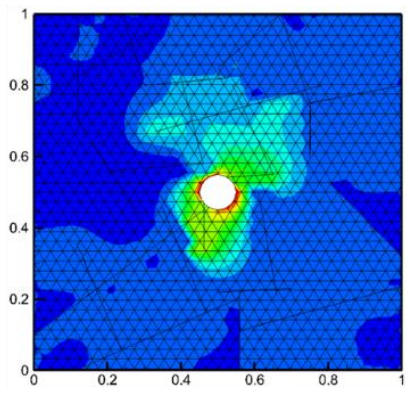

7000 s

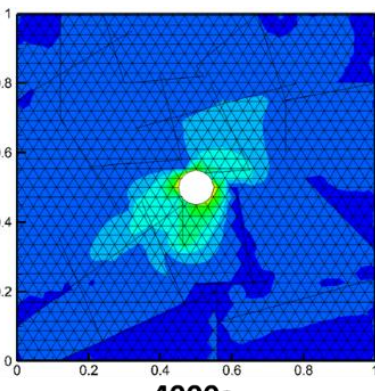

4000s

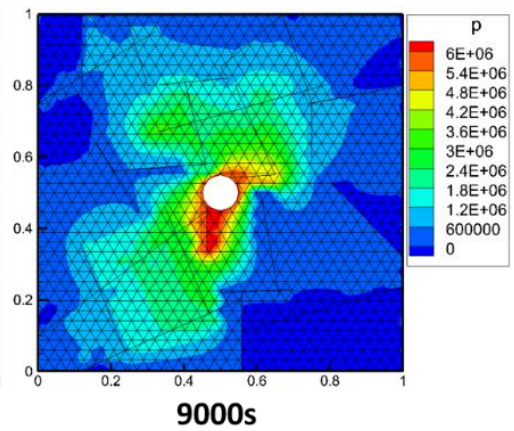

Figure 26 Simulated evolution of the injection-induced fluid pressure ( $\mathrm{Pa})$

\section{Conclusions}

In this study, we developed a new numerical manifold method model for analysis of fully coupled hydro-mechanical processes in porous rock with discrete fractures. In this model the porous rock and the fractures are both deformable and fluid conductive with large contrast of mechanical and hydraulic properties. On NMM two-cover-mesh system, global approximation for mechanical and fluid flow analysis are defined. Fractures are conveniently discretized by dividing the mathematical cover along fracture traces to physical cover resulting in a discontinuous model on a non-conforming mesh.

In this model, discrete fracture deformation (e.g. open and slip) and fracture fluid flow within a permeable and deformable porous rock matrix are thoroughly considered. For direct coupling in porous rock, an energy-work based scheme was used to link mechanical to fluid flow analysis with "work" as a bridge. For the discrete, complexly intersected fractures, a discontinuous model was developed for fracture deformation and fluid flow. For mechanical analysis, fractures may be in open, closed or sliding states. We revised Shi's original scheme [20] by considering fracture constitutive model for mechanically open states. For fluid flow in fractures, a previously developed model [29] was used considering along-fracture and normal-to-fracture fracture flow without introducing additional DOFs. When the mechanical aperture of a fracture is changing due to continuous opening or the change of contact states, its hydraulic aperture and hydraulic conductivity is updated. 
At the same time, under the effect of deformation, fluid flow and their coupling, the contact state may dynamically change, and the corresponding contact constraint is updated each time step. Open-close iterations are run each step until convergence of the correct contact state is achieved and the corresponding constraint is accurately enforced in the global equations. Therefore, indirect coupling is realized under stringent considerations of coupled HM effects and fracture constitutive behavior transfer dynamically.

We first verified the new model for coupled hydro-mechanical processes in rock with a single fracture, achieving the theoretical solution. Then we simulated several other scenarios of examples involving one and two sets of fractures. Last we applied the new model to calculate coupled HM behavior with complex fractures induced by loading and injection, and obtain reasonable results. This NMM model will be extended to analyzing large-scale hydraulic fracturing and fracture network stimulations in energy production.

\section{Acknowledgments}

This research was supported by the US Department of Energy to LBNL under contract No.DE-AC02-05CH11231.

\section{References}

[1] Rutqvist J, Stephansson O (2003) The role of hydromechanical coupling in fractured rock engineering. Hydrogeology Journal 11(1):7-40.

[2] Wang HF (2000) Theory of linear poroelasticity. Princeton University Press.

[3] Biot MA. General theory of three dimensional consolidation (1941) J Appl Phys 12: $155-164$.

[4] Noorishad J, Ayatollahi MS, Witherspoon PA (1982) Coupled stress and fluid flow analysis of fractured rock. Int J Rock Mech Min Sci 19:185- 193.

[5] Rutqvist J, Noorishad J, Tsang, CF (1998) Determination of fracture storativity in hard rocks using high-pressure injection testing. Water Resources Research 34(10):2551- 2560.

[6] Rutqvist J, Tsang CF, Stephansson O (2000) Uncertainty in the maximum principal stress estimated from hydraulic fracturing measurements due to the presence of the induced fracture. Int J Rock Mech Min Sci 37: 107-120.

[7] Witherspoon PA, Wang JSY, Iwai K, Gale JE (1980) Validity of the cubic law for fluid flow in a deformable fracture, Water Resour. Res. 16: 1016-1024.

[8] Rutqvist J, Borgesson L, Chijimatsu M, Jing L, Nguyen ST, Noorishad J, Tsang C-F (2001) Thermohydromechanics of partially saturated geological media: governing equations and formulation of four finite element models. Int J Rock Mech Min Sci 38(1):105-127.

[9] Noorishad J, Tsang CF, Witherspoon PA (1992) Theoretical and field studies of coupled hydromechanical behavior of fractured rock-1. Development and verification of a numerical simulator. Int J Rock Mech Min Sci 29(4):401- 409.

[10] Nguyen TS, Selvadurai APS (1995) Coupled thermal-hydrological- mechanical 
processes in sparsely fractured rock. Int J Rock Mech Min Sci Geomech Abstr 32: 465-480.

[11] Bower KM, Zyvoloski G (1997) A numerical model for thermo-hydro-mechanical coupling in fractured rock. Int J Rock Mech Min Sci Geomech Abstr 34: 1201-1211.

[12] Kohl T, Hopkirk RJ (1995) The finite element program "FRACTure" for the simulation of hot dry rock reservoir behavior. Geother-mics 24:345-359.

[13] Itasca Consulting Group 2011 UDEC Manual: Universal Distinct Element Code version 5.0 Minneapolis, MN, USA.

[14] Itasca Consulting Group (2013) 3DEC (Advanced, Three Dimensional Distinct Element Code), Version 5.0, Minneapolis, MN, USA.

[15] Kim Y, Amadei B, Pan E (1999) Modeling the effect of water, excavation sequence and rock reinforcement with discontinuous deformation analysis. Int J Rock Mech Min Sci 36(7): 949-970.

[16] Jing L, Ma Y, Fang Z (2001) Modeling of fluid flow and solid deformation for fractured rocks with discontinuous deformation analysis (DDA) method. Int J Rock Mech Min Sci 38(3): 343-355.

[17] Lamb AR, Gorman GJ, Elsworth D (2013) A fracture mapping and extended finite element scheme for coupled deformation and fluid flow in fractured porous media. International Journal for Numerical and Analytical Methods in Geomechanics 37: 2916-2936.

[18] Silvestre JR, Vargas EA, Vaz LE, Soares AC (2015) Modelling of coupled fluid-mechanical problems in fractured geological media using enriched finite elements. International Journal for Numerical and Analytical Methods in Geomechanics 39:1104- 1140.

[19] An X, Fu G, Ma G (2012) A comparison between the NMM and the XFEM in discontinuity modeling, Int. J. Comput. Methods 9(2): 1240030-1.

[20] Shi G (1992) Manifold method of material analysis. Transaction of the $9^{\text {th }}$ army conference on applied mathematics and computing, U.S. Army Research Office.

[21] Shi G (1996) Simplex integration for manifold method, FEM and DDA. Discontinuous Deformation Analysis (DDA) and Simulations of Discontinuous Media. TSI press. 205-262.

[22] Chen G, Ohnishi Y, Ito T (1998) Development of High-order Manifold Method. International Journal for Numerical Methods in Engineering 43: 685-712.

[23] Zheng $H, X u$ D (2014) New strategies for some issues of numerical manifold method in simulation of crack propagation. Int. J. Numer. Meth. Engng 97: 986-1010.

[24] Ning Y, An X, Ma G (2011) Footwall slope stability analysis with the numerical manifold method. Int J Rock Mech Min Sci 48: 964- 975.

[25] He L, An X, Ma G, Zhao Z (2013) Development of three-dimensional numerical manifold method for jointed rock slope stability analysis. Int J Rock Mech Min Sci 64: 22- 35.

[26] Wang Y, Hu M, Zhou Q, Rutqvist J (2014) Energy-work-based numerical manifold seepage analysis with an efficient scheme to locate the phreatic surface. 
International Journal for Numerical and Analytical Methods in Geomechanics 38: 1633-1650.

[27] Zheng H, Liu F, Li C (2015) Primal mixed solution to unconfined seepage flow in porous media with numerical manifold method. Applied Mathematical Modelling 39(2): 794-808.

[28] Hu M, Wang Y, Rutqvist J (2015) On continuous and discontinuous approaches for modeling groundwater flow in heterogeneous media using the Numerical Manifold Method: Model development and comparison. Advances in Water Resources 80: 17-29.

[29] Hu M, Rutqvist J, Wang Y (2016) A Practical Model for Flow in Discrete-Fracture Porous Media by Using the Numerical Manifold Method. Advances in water resources 97: 38-51.

[30] Zhang Q, Lin S, Xie Z, Su H (2016) Fractured porous medium flow analysis using numerical manifold method with independent covers. Journal of Hydrology 542: 790-808.

[31] Zhang H, Zhou L (2006) Numerical manifold method for dynamic nonlinear analysis of saturated porous media. International Journal for Numerical and Analytical Methods in Geomechanics 30: 927-951.

[32] Hu M, Wang Y, Rutqvist J (2016). Fully coupled hydro-mechanical numerical manifold modeling of porous rock with dominant fractures. Acta Geotechnica. DOI: 10.1007/s11440-016-0495-z.

[33] Munkres JR (1991) Analysis on manifolds. Addison-Wesley Publishing Company, Redwood City. 